\title{
OVERGROUPS OF REGULAR UNIPOTENT ELEMENTS IN SIMPLE ALGEBRAIC GROUPS
}

\author{
GUNTER MALLE AND DONNA M. TESTERMAN
}

\begin{abstract}
We investigate positive-dimensional closed reductive subgroups of almost simple algebraic groups containing a regular unipotent element. Our main result states that such subgroups do not lie inside proper parabolic subgroups unless possibly when their connected component is a torus. This extends the earlier result of Testerman and Zalesski treating connected reductive subgroups.
\end{abstract}

\section{INTRODUCTION}

Let $G$ be a simple linear algebraic group defined over an algebraically closed field. The regular unipotent elements of $G$ are those whose centraliser has minimal possible dimension (the rank of $G$ ) and these form a single conjugacy class which is dense in the variety of unipotent elements of $G$. The main result of our paper is a contribution to the study of positive-dimensional subgroups of $G$ which meet the class of regular unipotent elements. Since any parabolic subgroup must contain representatives from every unipotent conjugacy class, the question arises only for reductive, not necessarily connected subgroups, where we establish the following:

Theorem 1. Let $G$ be a simple linear algebraic group over an algebraically closed field, $X \leq G$ a closed reductive subgroup containing a regular unipotent element of $G$. If $\left[X^{\circ}, X^{\circ}\right] \neq 1$, then $X$ lies in no proper parabolic subgroup of $G$.

In addition, we show that for many simple groups $G$, there exists a closed reductive subgroup $X \leq G$ with $X^{\circ} \neq 1$ a torus and such that $X$ meets the class of regular unipotent elements of $G$. (See Proposition 7.2 and Examples 7.7, 7.11) Finally, we go on to consider subgroups of non-simple almost simple algebraic groups $G$ where there is a well-defined notion of regular unipotent elements in unipotent cosets of $G^{\circ}$. We establish the corresponding result in this setting; see Corollary 6.2 .

Received by the editors June 23, 2020, and, in revised form, January 9, 2021.

2020 Mathematics Subject Classification. Primary 20G05, 20G07, 20E28.

Key words and phrases. Regular unipotent elements, disconnected subgroups, reductive subgroups, almost simple linear algebraic groups.

Donna Testerman is the corresponding author.

Work on this article was begun while the authors were visiting the Mathematical Sciences Research Institute in Berkeley, California in Spring 2018 for the programme "Group Representation Theory and Applications" supported by the National Science Foundation under Grant No. DMS-1440140. The second author was supported by the Fonds National Suisse de la Recherche Scientifique grant number 200021-175571. We thank the Isaac Newton Institute for the Mathematical Sciences, where this work was completed, for support and hospitality during the programme "Groups, Representations and Applications: New Perspectives". This work was supported by: EPSRC grant number EP/R014604/1. 
The investigation of the possible overgroups of regular unipotent elements in simple linear algebraic groups has a long history. The maximal closed positivedimensional reductive subgroups of $G$ which meet the class of regular unipotent elements were classified by Saxl and Seitz [17] in 1997. In earlier work, see 21, Thm 1.9], Suprunenko obtained a particular case of their result. In order to derive from the Saxl-Seitz classification an inductive description of all closed positivedimensional reductive subgroups $X \leq G$ containing regular unipotent elements, one needs to exclude that any of these can lie in proper parabolic subgroups. For connected $X$ this was shown by Testerman and Zalesski in [22, Thm 1.2] in 2013. They then went on to determine all connected reductive subgroups of simple algebraic groups which meet the class of regular unipotent elements. Our result generalises [22, Thm 1.2] to the disconnected case and thus makes the inductive approach possible. It is worth pointing out that the analogous result is no longer true even for simple subgroups once one relaxes the condition of positive-dimensionality. For example, there exist reducible indecomposable representations of the group $\mathrm{PSL}_{2}(p)$ whose image in the corresponding $\mathrm{SL}(V)$ contains a matrix with a single Jordan block, i.e., the image meets the class of regular unipotent elements in $\mathrm{SL}(V)$. In [3], Burness and Testerman consider $\mathrm{PSL}_{2}(p)$-subgroups of exceptional type simple algebraic groups which meet the class of regular unipotent elements and show that with the exception of two precise configurations, such a subgroup does not lie in a proper parabolic subgroup of $G$ (see [3, Thms 1 and 2]).

Our proof of Theorem[1]relies on the result of Testerman-Zalesski [22] in the connected case, which actually implies our theorem in characteristic 0 (see Remark 2.1) as well as on results of Saxl-Seitz [17] classifying almost simple irreducible and tensor indecomposable subgroups of classical groups containing regular unipotent elements and maximal reductive subgroups in exceptional groups with this property. For the exceptional groups we also use information on centralisers of unipotent elements and detailed knowledge of Jordan block sizes of unipotent elements acting on small modules, as found in Lawther [6]. For establishing the existence of positivedimensional reductive subgroups $X \leq G$, with $X^{\circ}$ a torus, and $X$ meeting the class of regular unipotent elements, we produce subgroups which centralise a non-trivial unipotent element and hence necessarily lie in a proper parabolic subgroup of $G$. (See [15, Thm 17.10, Cor. 17.15].)

After collecting some useful preliminary results we deal with the case of $G=$ $\mathrm{SL}(V)$ in Section 3 with the orthogonal case in Section 4 , and with the simple groups of exceptional type in Section 5 The case of almost simple groups is deduced from the connected case in Corollary 6.2 Finally, in Section 7 we discuss the case when $X^{\circ}$ is a torus.

\section{Preliminary Results}

In this paper we consider almost simple algebraic groups defined over an algebraically closed field $k$ of characteristic $p \geq 0$ and investigate closed positivedimensional subgroups that contain a regular unipotent element. For us, throughout "algebraic group" will mean "linear algebraic group", and all vector spaces will be finite-dimensional vector spaces over $k$. An algebraic group $G$ is called an almost simple algebraic group if $G^{\circ}$ is simple and $G / Z\left(G^{\circ}\right)$ embeds into Aut $\left(G^{\circ}\right)$. Thus, $G$ is an extension of $G^{\circ}$ by a subgroup of its group of graph automorphisms (see, e.g., [15, Thm 11.11]). As a matter of convention, a "reductive subgroup" 
of an algebraic group will always mean a closed subgroup whose unipotent radical is trivial. In particular, a reductive group may be disconnected. For an algebraic group $H$, we write $R_{u}(H)$ to denote the unipotent radical of $H$. Throughout, all $k G$-modules are rational, as are all extensions, and cohomology groups are those associated to rational cocycles.

Let us point out that for the question treated here, the precise isogeny type of the ambient simple algebraic group $G^{\circ}$ will not matter, as isogenies preserve parabolic subgroups as well as regular unipotent elements. (If $G$ is almost simple and $p$ does not divide the order of the fundamental group of $G^{\circ}$, the natural map $G \rightarrow G / Z\left(G^{\circ}\right)$ induces an isogeny of $G^{\circ}$ onto its adjoint quotient, preserving regular unipotent elements in $G$; in the general case, a reduction to $G^{\circ}$ of adjoint type is given in [18, I.1.7].) In particular, for $G$ a classical type simple algebraic group we will argue for the groups $\mathrm{SL}(V), \mathrm{Sp}(V)$ and $\mathrm{SO}(V)$, and for the groups of type $B_{l}$ and $C_{l}$ defined over $k$ of characteristic 2, we may choose to work with whichever group is more convenient under the given circumstances.

We start by making two useful observations which will simplify the later analysis.

Remark 2.1. In the situation of Theorem 1 assume that $p=0$. As $\left|X: X^{\circ}\right|$ is finite, some power of a regular unipotent element $u \in X$ will lie in $X^{\circ}$. In characteristic 0 any power of a regular unipotent element is again regular unipotent, so here we are thus reduced to studying the connected reductive subgroup $X^{\circ}$ satisfying the same assumptions. In that case, the conclusion of Theorem 1 was established in [22, Thm 1.2]. Hence, in proving Theorem 1 we may assume $p>0$ whenever convenient. Furthermore, we will assume without loss of generality that $X=X^{\circ}\langle u\rangle$.

Remark 2.2. Let $X=X^{\circ}\langle u\rangle$ be a reductive subgroup of a connected reductive group $G$ such that $u$ is regular unipotent in $G$ and $\left[X^{\circ}, X^{\circ}\right] \neq 1$. Let $X_{1}$ be one of the simple components of $\left[X^{\circ}, X^{\circ}\right]$ and set $H:=\left\langle X_{1}, u\right\rangle$. Then $H^{\circ}=\prod_{i} X_{1}^{u^{i}}$, so $\langle u\rangle$ acts transitively on the set of simple components of $H^{\circ}$, and if $X$ lies in a proper parabolic subgroup of $G$, then so does $H$. Thus, when proving Theorem 1 we may as well assume that the simple components of $X^{\circ}$ are permuted transitively by $u$.

2.1. Jordan forms and tensor products. The following elementary fact will be used throughout (see also [17, Lemma 1.3(i)]):

Lemma 2.3. Assume that $p>0$ and let $u \in \mathrm{SL}(V)$ be unipotent with a single Jordan block. Write $\operatorname{dim} V=a p+b$ with $0 \leq b<p$. Then $u^{p}$ has $p$ Jordan blocks, $b$ of size $a+1$ and the other $p-b$ of size $a$.

Lemma 2.4. Let $u \in \mathrm{SL}(V)$ be a unipotent element with a single Jordan block of size $n=\operatorname{dim} V$, or with two Jordan blocks of sizes $n-1,1$ or $n-2,2$. If u preserves the factors in a non-trivial tensor product decomposition of $V$ then $\operatorname{dim} V=4$ and $u$ has two Jordan blocks on $V$. If $p=2$ these are of sizes 2,2 .

Proof. Using the description of Jordan block sizes of unipotent elements in tensor products given in [17. Lemma 1.5] we see that necessarily $\operatorname{dim} V=4$ and either $u$ has Jordan block sizes 2,2 , or $p \neq 2$ and $u$ has Jordan block sizes 3,1 , as claimed. 
Before establishing a useful consequence of Lemma 2.4, we recall the following well-known Clifford-theoretic result, see, e.g., [2, Prop. 2.6.2]:

Lemma 2.5. Let $N \unlhd H$ be groups with $H / N$ finite cyclic and $V$ be a finitedimensional irreducible $k H$-module. Then $\left.V\right|_{N}=\bigoplus_{g} U^{g}$, where $U$ is any irreducible $k N$-submodule of $V$ and $g$ runs over a system of coset representatives of the stabiliser of $U$ in $H$. Moreover, the $U^{g}$ are pairwise non-isomorphic $k N$-modules.

We now show the desired corollary of Lemma 2.4.

Lemma 2.6. Let $H \leq \mathrm{SL}(V)$ be connected reductive with non-trivial derived subgroup and assume that $\left.V\right|_{H}$ is completely reducible and homogeneous. If $H$ is normalised by a unipotent element $u \in \mathrm{SL}(V)$ with a single Jordan block of size $n=\operatorname{dim} V$, or with two Jordan blocks of sizes $n-1,1$ or $n-2,2$ then either $V$ is an irreducible $H$-module, or $\operatorname{dim} V=4, H\langle u\rangle$ preserves a non-trivial tensor product decomposition of $V$, and $u$ has two Jordan blocks on $V$, of sizes 2 , 2 if $p=2$.

Proof. Let $u \in \mathrm{SL}(V)$ be the unipotent element normalising $H$ as in the assumption. Let $V_{1} \leq V$ be an irreducible $H\langle u\rangle$-submodule of $V$. As $V$ is homogeneous as an $H$-module, Lemma 2.5 shows that $\left.V_{1}\right|_{H}$ is irreducible. Since $[H, H] \neq 1$, we have $\operatorname{dim} V_{1}>1$. Then with $W=\operatorname{Hom}_{H}\left(V_{1}, V\right)$ we have $V \cong V_{1} \otimes W$ as an $H$-module, and this decomposition is stabilised by $u$ (see e.g. [15, Prop. 18.1]). Applying Lemma 2.4, this implies that either $\operatorname{dim} W=1$, whence $V=V_{1}$ is irreducible for $H$, or we are in the exceptional case of that result, as in the conclusion.

The proof of the next result is modelled after the proof of [17, Prop. 2.1] which treats a more special situation:

Lemma 2.7. Assume $p>0$ and let $X=X^{\circ}\langle u\rangle \leq \mathrm{SL}(V)$ be a reductive subgroup and $u \in \operatorname{SL}(V)$ a unipotent element with a single Jordan block of size $n:=\operatorname{dim} V>$ 1 , or with two Jordan blocks of sizes $n-1,1$ or $n-2,2$. If $X^{\circ}$ acts irreducibly on $V$, then either $\left[X^{\circ}, X^{\circ}\right]$ is simple, or one of the following holds:

(1) $\operatorname{dim} V=4, X$ preserves a non-trivial tensor decomposition of $V$ and $u$ has two Jordan blocks on $V$. If $p=2$ these are of size 2,2 ;

(2) $p \in\{2,3\}, V=V_{1} \otimes \cdots \otimes V_{p}$ as an $X^{\circ}$-module with $\operatorname{dim} V_{i}=2,\left[X^{\circ}, X^{\circ}\right]=$ $A_{1}^{p}$, u permutes both sets of factors transitively and has a single Jordan block on $V$. Moreover, $u^{p}$ has a single Jordan block on each $V_{i}$; or

(3) $p=2, V=V_{1} \otimes V_{2}$ as an $X^{\circ}$-module with $\operatorname{dim} V_{i}=3$, u has Jordan blocks of sizes 8, 1 on $V$ and $u^{2}$ has a single Jordan block on each $V_{i}$.

Here, in (2) and (3), $X$ does not preserve the stated tensor product decomposition of $V$.

Proof. Note that $\left[X^{\circ}, X^{\circ}\right] \neq 1$ as $\operatorname{dim} V>1$ and $X^{\circ}$ acts irreducibly. Write $\left[X^{\circ}, X^{\circ}\right]=X_{1} \cdots X_{s}$ with simple algebraic groups $X_{i}$, so $V=V_{1} \otimes \cdots \otimes V_{s}$ with non-trivial irreducible $X_{i}$-modules $V_{i}$. Now $u$ permutes the factors $X_{i}$ and their corresponding tensor factors $V_{i}$. Assume that $s>1$. If $u$ has at least two orbits on the set of $X_{i}$, this yields a corresponding $u$-invariant tensor decomposition of $V$. By Lemma 2.4 we reach case (1).

Henceforth, we may assume that $u$ permutes the $X_{i}$, and thus the $V_{i}$, transitively. In particular all $V_{i}$ have the same dimension $m$, that is, $\operatorname{dim} V=m^{s}$, and $s=: p^{a}>$ 1 is a power of $p$. Let $b$ be minimal with $p^{b} \geq m$. Now, $u^{p^{a}}$ stabilises all $V_{i}$, so 
is a tensor product of matrices of size $m$ and thus of order at most $p^{b}$. Hence $|u|$ divides $p^{a+b}$. On the other hand,

$$
p^{a+b} \geq|u| \geq \operatorname{dim} V-2=m^{s}-2=m^{p^{a}}-2>p^{(b-1) p^{a}}-2 .
$$

The above conditions imply that either $a=b=1, m=2$ and $s=p \leq 3$, or $a=1$, $b=s=p=2$ and $m=3$.

In the first case, $\operatorname{dim} V=m^{s}=2^{p}, p \leq 3$, and as $m=2$ all simple factors $X_{i}$ of $\left[X^{\circ}, X^{\circ}\right]$ must have type $A_{1}$, as in (2). The statement about the Jordan form of $u$ follows from Lemma 2.3 .

In the second case we have $\operatorname{dim} V=9$, and our inequalities force that $|u|=8$ and hence $u$ has Jordan blocks of sizes 8,1 or 7,2 and by Lemma 2.3, $u^{2}$ has Jordan blocks of sizes $4,4,1$, respectively $4,3,1,1$. The latter cannot arise as the block sizes of a tensor product of two $3 \times 3$ unipotent matrices by [17, Lemma 1.5], so we are in the former case and $u^{2}$ has a single Jordan block on each $V_{i}$, as in (3).

2.2. On subgroups containing regular unipotent elements. For connected groups, the following result from [22, Lemma 2.6] will be useful:

Lemma 2.8. Let $G$ be connected reductive, $P \leq G$ a parabolic subgroup with Levi complement $L$ and assume that $u \in P$ is regular unipotent in $G$. Then the image of $u$ is regular unipotent in $L$ and hence in each simple factor of $[L, L]$.

Lemma 2.9. Let $G$ be simple and $H \leq G$ be a connected reductive subgroup normalised by a regular unipotent element $u$ of $G$. Assume that $H=Y_{1} \circ Y_{2}$ is a central product with $Y_{1} \neq 1$ such that $u$ acts by an inner automorphism on $Y_{1}$. Then $Y_{1}=H$ contains a regular unipotent element of $G$.

Proof. By assumption, $u$ acts as an inner automorphism on $Y_{1}$, say by $z \in Y_{1}$. Thus, $u z^{-1}$ and $Y_{2}$ are contained in $C_{H}\left(Y_{1}\right)$ and so $u \in H\langle u\rangle=\left(Y_{1} Y_{2}\right)\langle u\rangle \leq Y_{1} \circ C_{H}\left(Y_{1}\right)$. But then [22, Prop. 2.3] implies that $Y_{2}=1$. Now $u=z \cdot u z^{-1}$ is regular unipotent. Replacing $u z^{-1}$ and $z$ by their unipotent parts respectively, we may assume both to be unipotent and lying in a common Borel subgroup of $G$. As $u z^{-1}$ centralises $Y_{1}$ and thus isn't regular, $z \in Y_{1}$ must be regular by [22, Lemma 2.4].

Lemma 2.10. Let $G$ be simple and $H<G$ a connected reductive subgroup containing a regular unipotent element $u$ of $G$. Then $u$ is regular in $H$.

Proof. Let $B<H$ be a Borel subgroup of $H$ containing $u$. Assume $u$ is not regular unipotent in $H$. By [19, Ch. III, 1.13] it may be written as a product of root elements

$$
u=\prod_{\alpha} u_{\alpha}(1) \prod_{\beta} u_{\beta}\left(c_{\beta}\right) \quad \text { for suitable } c_{\beta} \in k,
$$

where the first product runs over a proper subset of the simple roots of the root system $\Phi$ of $H$ with respect to the pair $(T, B)$ where $T<B$ is some maximal torus, and the second one over the roots in $\Phi^{+}$of height at least 2 . Thus, $u$ lies in the unipotent radical of the parabolic subgroup of $H$ whose Levi factor is generated by the root subgroups for the simple roots not occurring in the representation of $u$ and their negatives, which thus is not a torus.

By Borel-Tits, $u$ then also lies in the unipotent radical of a proper parabolic subgroup $P$ of $G$ with non-toral Levi factor. But then, when writing $u$ as a product of root elements for $G$ with respect to a Borel subgroup contained in $P$, not all 
simple roots can occur, whence $u$ is not regular in $G$. This contradiction achieves the proof.

We will make frequent use of the following result, the second part of which was essentially shown by Saxl and Seitz [17, Prop. 2.2]:

Proposition 2.11. Let $X=X^{\circ}\langle u\rangle$ be a reductive subgroup of the simple classical group $G=\mathrm{SL}(V), \operatorname{Sp}(V)$ or $\mathrm{SO}(V)$ with $X^{\circ}$ simple and irreducible on $V$, where $\operatorname{dim} V \geq 7$ when $G$ is of orthogonal type. If $X$ contains a regular unipotent element $u$ of $G$, then $X^{\circ}$ acts tensor indecomposably on $V$.

Furthermore, either $X=B_{3}<G=D_{4}$, or $X$ and the highest weight of $X^{\circ}$ on $V$ are as in Table 1 (up to Frobenius twists and taking duals) and $u$ has a single Jordan block.

TABLE 1. Simple modules with regular unipotent elements. The last column records (an upper bound for) the order of a regular unipotent element $u \in X$.

\begin{tabular}{c|cccc}
$X$ & $\lambda$ & $\operatorname{dim} V$ & cond. & $|u|$ \\
\hline$A_{1}$ & $m \varpi_{1}$ & $m+1$ & $m<p$ & $p$ \\
$A_{l}$ & $\varpi_{1}$ & $l+1$ & $l>1$ & $<p(l+1)$ \\
$B_{l}$ & $\varpi_{1}$ & $2 l+1$ & $p>2$ & $<p(2 l+1)$ \\
$C_{l}$ & $\varpi_{1}$ & $2 l$ & & $<2 p l$ \\
$G_{2}$ & $\varpi_{1}$ & 7 & $p>2$ & $\leq p^{2}$ \\
$G_{2}$ & $\varpi_{1}$ & 6 & $p=2$ & 8 \\
$A_{2} .2$ & $\varpi_{1}+\varpi_{2}$ & 8 & $p=2$ & 8 \\
$D_{l} .2$ & $\varpi_{1}$ & $2 l$ & $p=2, l \geq 3$ & $<4 l$
\end{tabular}

Proof. Assume that $V=V_{1} \otimes V_{2}$ for non-trivial irreducible $X^{\circ}$-modules $V_{i}$. If $X=X^{\circ}$, then Lemma 2.4 gives that $\operatorname{dim} V=4$ and $G=D_{2}$, but this is not simple. If some power $u^{j}$ acts as an inner element $y$ on $X^{\circ}$, then $u^{j} y^{-1}=z \in X$ centralises $X^{\circ}$, hence, as $X^{\circ}$ acts irreducibly, we must have that $u^{j}$ equals the unipotent part of $y$ and so lies in $X^{\circ}$. So now we may assume that $u \notin X^{\circ}$ and hence $X=A_{l} .2$ $(l \geq 2), D_{l} .2(l \geq 4)$ or $E_{6} .2$, with $p=2$, or $X=D_{4} .3$ and $p=3$. Unipotent elements in $X=E_{6} .2$ have order at most 32 , but there is no faithful representation of $X$ of dimension less than 54 (the 27-dimensional modules for $E_{6}$ are not invariant under the graph automorphism). Thus $X^{\circ}$ is of classical type. Let $d$ denote the dimension of its natural module. Then, e.g., by [12, Tab. 2] we have $\operatorname{dim} V_{i} \geq d$, so $\operatorname{dim} V \geq d^{2}$, but unipotent elements of $X$ have order less than $p^{2} d$. Thus $d<p^{2}$. Note that $D_{4}$ with $p=3$ cannot occur, as here unipotent elements have order at most $27<d^{2}-2=62$ (see Lemma 2.12). This only leaves the possibility $X^{\circ}=A_{2}, p=2$, and $\operatorname{dim} V=9$. But there is no 9-dimensional irreducible orthogonal module in characteristic 2 , so in fact $V$ must be tensor indecomposable for $X^{\circ}$. The remaining assertions are now shown in [17, Prop. 2.2].

We will obtain a similar classification for unipotent elements in $\operatorname{SL}(V)$ with a Jordan block of size $\operatorname{dim} V-1$ when $p=2$ in Proposition 4.1 . 
2.3. Jordan forms and orders of regular unipotent elements. While the notion of regular unipotent element is well known for connected reductive groups, this is much less so for non-connected reductive groups. Still, similar results hold.

Let $G$ be a not necessarily connected reductive algebraic group and let $x \in G$ be unipotent. Spaltenstein [18, p. 41 and II.10.1] has shown (generalising a result of Steinberg in the connected case) that the coset $x G^{\circ}$ of the connected component $G^{\circ}$ contains a unipotent $G^{\circ}$-conjugacy class $C$ that is dense in the variety of unipotent elements of $x G^{\circ}$, called the class of regular unipotent elements of $x G^{\circ}$. Since this variety is irreducible [18, Cor. I.1.6], $C$ is also the unique class of unipotent elements in $x G^{\circ}$ of maximal dimension.

We now describe the Jordan block structure of regular unipotent elements in classical type almost simple groups on their natural representation. For us, the natural representation for the extension $A_{l-1} .2$ of $A_{l-1}, l \geq 3$, by its graph automorphism of order 2 is defined by its embedding into the stabiliser in $\mathrm{GO}_{2 l}$ of a pair of complementary totally singular subspaces, with $A_{l-1}$ acting in its natural representation, respectively its dual, on these subspaces. We do not consider $D_{4} .3$ or $D_{4} \cdot \mathfrak{S}_{3}$ as being of classical type.

Lemma 2.12. Let $G=G^{\circ}\langle u\rangle$ be almost simple of classical type with u regular unipotent in $u G^{\circ}$. Then in the natural representation of $G$ :

(a) $u$ has a single Jordan block for $G=A_{l}, B_{l}$ (when $p \neq 2$ ), $C_{l}$, and for $G=D_{l} .2$ when $p=2$;

(b) $u$ has two Jordan blocks of sizes $2 l, 1$ when $p=2$ for $G=B_{l}$;

(c) $u$ has two Jordan blocks of sizes $2 l-1,1$ when $p \neq 2$, respectively of sizes $2 l-2,2$ when $p=2$ for $G=D_{l}$;

(d) $u$ has a single Jordan block of size $2 l$ when $p=2$ for $G=A_{l-1} .2$ with $l$ odd; and

(e) $u$ has two Jordan blocks of sizes $2 l-2,2$ when $p=2$ for $G=A_{l-1} .2$ with $l$ even.

Proof. Only (d) and (e) are not shown in [17, Lemma 1.2]. Spaltenstein [18, I.2.7, I.2.8(c)] gives a description of the unipotent classes in $A_{l-1} .2 \backslash A_{l-1}$ in terms of the Jordan normal form of the square of the elements on the natural $A_{l-1}$-module, and a formula for the centraliser dimension. From this it can be seen that elements $u$ with minimal centraliser dimension are those for which $u^{2}$ has one Jordan block of size $l$ if $l$ is odd, and two blocks of sizes $l-1,1$ if $l$ is even. Thus, in the natural $2 l$-dimensional orthogonal representation of $A_{l-1} .2$, the element $u^{2}$ has two Jordan blocks of size $l$, respectively four of sizes $l-1, l-1,1,1$. Given the possible Jordan block shapes of unipotent elements in $D_{l} .2$ in its natural representation [18, I.2.6], the claim for $u$ follows with Lemma 2.3 .

Since the natural representations are faithful, the above result also allows one to read off the orders of regular unipotent elements of almost simple classical groups.

2.4. Some results on extensions. We conclude this preparatory section by collecting some basic properties on Ext-groups. We state the following well-known result for future reference (see [24, Prop. 3.3.4]). 
Lemma 2.13. Let $H$ be a group, and $V_{i}, U_{j}$ with $1 \leq i \leq n, 1 \leq j \leq m$, be $k H$-modules. Then

$$
\operatorname{Ext}_{H}^{1}\left(\bigoplus_{i} V_{i}, \bigoplus_{j} U_{j}\right) \cong \bigoplus_{i, j} \operatorname{Ext}_{H}^{1}\left(V_{i}, U_{j}\right)
$$

We thank Jacques Thévenaz for pointing out the following result:

Lemma 2.14. Let $k$ be a field, $N \unlhd H$ be groups and $U, V$ two finite-dimensional $k H$-modules on which $N$ acts trivially. Assume that $\operatorname{Ext}_{N}^{1}(k, k)=0$. Then

$$
\operatorname{Ext}_{H / N}^{1}(U, V) \cong \operatorname{Ext}_{H}^{1}(U, V) .
$$

Proof. We use the (exact) inflation-restriction sequence for cohomology (see [24, 6.8.3]) for a $k H$-module $M$ :

$$
0 \rightarrow H^{1}\left(H / N, M^{N}\right) \stackrel{\text { inf }}{\longrightarrow} H^{1}(H, M) \stackrel{\text { res }}{\longrightarrow} H^{1}(N, M)^{H / N} \rightarrow \ldots
$$

which by [24, 6.1.2] can be interpreted as the Ext-sequence

$$
0 \rightarrow \operatorname{Ext}_{H / N}^{1}\left(k, M^{N}\right) \rightarrow \operatorname{Ext}_{H}^{1}(k, M) \rightarrow \operatorname{Ext}_{N}^{1}(k, M)^{H / N} \rightarrow \ldots
$$

Applying this with $M=U^{*} \otimes V$ and using $\operatorname{Ext}^{1}\left(k, U^{*} \otimes V\right) \cong \operatorname{Ext}^{1}(U, V)$ (see [1, Cor. 1]) the previous sequence becomes

$$
0 \rightarrow \operatorname{Ext}_{H / N}^{1}\left(k,\left(U^{*} \otimes V\right)^{N}\right) \rightarrow \operatorname{Ext}_{H}^{1}(U, V) \rightarrow \operatorname{Ext}_{N}^{1}(U, V)^{H / N} \rightarrow \ldots
$$

As $N$ acts trivially on $U$ and $V$, the first term equals $\operatorname{Ext}_{H / N}^{1}\left(k, U^{*} \otimes V\right) \cong$ $\operatorname{Ext}_{H / N}^{1}(U, V)$, while the third is $\operatorname{Ext}_{N}^{1}\left(k^{\operatorname{dim} U}, k^{\operatorname{dim} V}\right)^{H / N}=0$ by our hypothesis on $\operatorname{Ext}_{N}^{1}(k, k)$ and Lemma 2.13, whence exactness of the sequence implies our claim.

Lemma 2.15. $\quad$ (a) Let $H$ be a semisimple algebraic group. Then there are no non-trivial self-extensions between irreducible $H$-modules.

(b) Let $H_{1} \circ H_{2}$ be a semisimple group acting on $V_{1} \oplus V_{2}$, with $H_{i}$ acting trivially on $V_{3-i}$. Then

$$
H^{1}\left(H_{1} H_{2}, V_{1} \oplus V_{2}\right) \cong H^{1}\left(H_{1}, V_{1}\right) \oplus H^{1}\left(H_{2}, V_{2}\right) .
$$

Proof. Part (a) is [4, II.2.12(1)]. In (b) by Lemma 2.13 we have

$$
H^{1}\left(H_{1} H_{2}, V_{1} \oplus V_{2}\right) \cong H^{1}\left(H_{1} H_{2}, V_{1}\right) \oplus H^{1}\left(H_{1} H_{2}, V_{2}\right) \text {. }
$$

As $H_{2}$ acts trivially on $V_{1}$, the first summand is isomorphic to

$$
H^{1}\left(H_{1} /\left(H_{1} \cap H_{2}\right), V_{1}\right) \cong H^{1}\left(H_{1}, V_{1}\right)
$$

by two applications of Lemma 2.14, and similarly for the second summand.

\section{THE CASE OF SL $(V)$}

In this section we prove Theorem 1 for those classical type simple algebraic groups for which regular unipotent elements have a single Jordan block on their natural module (see Lemma 2.12). We are in the following situation: $X \leq \mathrm{SL}(V)$ is a (not necessarily connected) reductive subgroup of the form $X=X^{\circ}\langle u\rangle$ for a regular unipotent element $u$ of $\operatorname{SL}(V)$. We also assume that $X^{\circ}$ is not a torus; this case will be considered in Section [7.1. We will show that $X$ cannot be contained in a proper parabolic subgroup of $\operatorname{SL}(V)$, that is, $X$ acts irreducibly on $V$. For this, we may whenever convenient, assume that $X^{\circ}$ is semisimple, since if $\left[X^{\circ}, X^{\circ}\right]\langle u\rangle$ is 
not contained in a proper parabolic subgroup of $\mathrm{SL}(V)$ then neither is $X^{\circ}\langle u\rangle$. As $u$ has a single Jordan block on $V$, if $V_{1}<V_{2} \leq V$ are $u$-invariant subspaces, then $u$ has a single Jordan block on $V_{2} / V_{1}$.

3.1. The completely reducible case. We first deal with the case when $V$ is completely reducible for $X^{\circ}$.

Lemma 3.1. Let $X \leq \mathrm{SL}(V)$ be a reductive subgroup of the form $X=X^{\circ}\langle u\rangle$ for a regular unipotent element $u$ of $\mathrm{SL}(V)$, such that $X^{\circ}$ is not a torus. Assume that $\left.V\right|_{X^{\circ}}$ is completely reducible. Then $V$ is an irreducible $X$-module.

Proof. Decompose $\left.V\right|_{X^{\circ}}=V_{1} \oplus \cdots \oplus V_{m}$ into its homogeneous components $V_{i}$. Then $\langle u\rangle$ acts on the set of components, transitively since otherwise we obtain a $u$ invariant decomposition of $V$, contradicting regularity of $u$. Thus $v:=u^{m}$ stabilises each component $V_{i}$, acting as a single Jordan block on it by Lemma 2.3, and $V_{i}$ is homogeneous as an $X^{\circ}$-module. Since $X^{\circ}$ is not a torus, Lemma 2.6 implies that $V_{i}$ is an irreducible $X^{\circ}$-module. As the $V_{i}$ are permuted transitively by $u$, the module $V$ is irreducible for $X$.

In view of Lemma 3.1, it seems interesting to determine the structure of irreducible subgroups containing regular unipotent elements.

Proposition 3.2. Assume $p>0$. Let $X \leq \mathrm{SL}(V)$ be a reductive subgroup of the form $X=X^{\circ}\langle u\rangle$ for a regular unipotent element $u$ of $\mathrm{SL}(V)$, such that $X^{\circ} \neq 1$ is semisimple. Assume that $X$ acts irreducibly on $V$. Then

- $\left.V\right|_{X^{\circ}}=V_{1} \oplus \cdots \oplus V_{m}$ for submodules $V_{i}$, transitively permuted by $u$, on which $u^{m}$ acts with a single Jordan block; and

- $X^{\circ}=Y_{1} \cdots Y_{r}$ for distinct semisimple normal subgroups $Y_{1}, \ldots, Y_{r}$ transitively permuted by $u$, with $r \mid m$, where $Y_{i}$ acts trivially on $V_{j}$ for $j \not \equiv i$ $(\bmod r)$, and in addition one of the following holds:

(1) the $Y_{i}$ are isomorphic simple algebraic groups and $V_{i}$ is an irreducible tensor indecomposable $Y_{i}(\bmod r)$-module, for $1 \leq i \leq m$; if $r<m$ then $p=2, Y_{i}=A_{l}$ for some $l \geq 2, \operatorname{dim} V_{i}=l+1$ and $m=2 r$; or

(2) $p \in\{2,3\}, m=r, Y_{i}=A_{1}^{p}$ for all $i$, with $u^{m}$ permuting the $p$ factors transitively, and each $V_{i}$, as a $Y_{i}$-module, is a tensor product of $p$ irreducible 2-dimensional $A_{1}$-modules.

Proof. As $V$ is irreducible for $X$, by Lemma 2.5, $\left.V\right|_{X^{\circ}}=V_{1} \oplus \cdots \oplus V_{m}$ is a direct sum of non-isomorphic irreducible $X^{\circ}$-modules permuted transitively by $u$, with $u\left(V_{i}\right)=V_{i+1}$ for all $i$. Thus $u^{m}$ stabilises each $V_{i}$ and acts with a single Jordan block on it by Lemma 2.3. The semisimple group $X^{\circ}$ is a product of simple normal subgroups. Let $Y_{1}$ denote the product of those simple factors of $X^{\circ}$ acting nontrivially on $V_{1}$. By Lemma 2.7 applied to $Y_{1}\left\langle u^{m}\right\rangle$ acting on $V_{1}$, either $Y_{1}$ is simple, and then it acts tensor indecomposably on $V_{1}$ by Proposition 2.11 or $p \in\{2,3\}$, $Y_{1}=A_{1}^{p}, u^{m}$ permutes the $p$ factors transitively, and $V_{1}$ is a tensor product of $p$ irreducible 2-dimensional $A_{1}$-modules.

Now $Y_{i+1}:=Y_{1}^{u^{-i}}$ acts non-trivially on $u^{i}\left(V_{1}\right)=V_{i+1}$ for $i=1, \ldots, m-1$. Thus $X^{\circ}$ is generated by, and hence equal to the product of the $Y_{i}$, which are permuted transitively by $u$. Since each $Y_{i}$ is an orbit of $u^{m}$ on the set of simple factors of $X^{\circ}$, these sets are mutually disjoint. Let $u^{r}$ be the smallest power of $u$ stabilising $Y_{1}$. Then $X^{\circ}=Y_{1} \cdots Y_{r}$ and $r \mid m$. 
Assume $Y_{i}$ is simple and $r \neq m$. As $u^{r}$ normalises $Y_{1}$ but does not stabilise $V_{1}$, it must act as an outer automorphism of the simple group $Y_{1}$. So $p \in\{2,3\}$ and moreover, $u^{r p}$ acts as an inner automorphism on $Y_{1}$ and stabilises the $Y_{1}$-module $V_{1}$. So $u^{r p} \leq\left\langle u^{m}\right\rangle$, and as $r \mid m$ this implies that $m=r p$. By Proposition 2.11 the group $Y_{1}\left\langle u^{m}\right\rangle$ acting on $V_{1}$ occurs in Table 1. If $p=3$, so $Y_{1}=D_{4}$, there is no such entry. So we have $p=2$. The case $D_{l} .2$ on the natural module does not occur here, as $V_{1}$ is not $u^{r}$-stable. So $Y_{1}=A_{l}$ with $l \geq 2, Y_{1}\left\langle u^{r}\right\rangle=A_{l} .2$ and $\operatorname{dim} V_{1}=\operatorname{dim} u^{r}\left(V_{1}\right)=l+1$ while $u^{2 r}=u^{m}$ stabilises all $V_{i}$, as in (1).

Finally, assume that $p \in\{2,3\}$ and $Y_{1}=A_{1}^{p}$. Then $Y_{1}$ is normalised by $u^{r}$, and $u^{m}$ permutes its $p$ factors transitively, where $r \mid m$. This forces $r=m$, as in (2).

Remark 3.3. In the situation of Proposition 3.2, for $1 \leq i \leq r$ set $W_{i}:=V_{i}$ if $m=r$, and $W_{i}:=V_{i} \oplus u^{r}\left(V_{i}\right)$ if $m=2 r$. Then $W_{i}$ is an irreducible $Y_{i}\left\langle u^{r}\right\rangle$-module and $Y_{i}$ acts trivially on $W_{j}$ for $j \neq i$. So $W_{i}^{\prime}:=\bigoplus_{j \neq i} W_{j}$ is the trivial $Y_{i}$-homogeneous component of $V$ and the decomposition $V=W_{i} \oplus W_{i}^{\prime}$ is stabilised by $u^{r}$. Moreover, $u^{r}$ acts by a single Jordan block on each $W_{i}$.

\subsection{The not completely reducible case.}

Lemma 3.4. Let $X \leq \mathrm{SL}(V)$ be a reductive subgroup of the form $X=X^{\circ}\langle u\rangle$ for a regular unipotent element $u$ of $\operatorname{SL}(V)$, with $\left[X^{\circ}, X^{\circ}\right] \neq 1$. Then $\operatorname{Soc}\left(\left.V\right|_{\left[X^{\circ}, X^{\circ}\right]}\right)$ is an irreducible $X$-module. That is, the socle series of $V$ as $\left[X^{\circ}, X^{\circ}\right]$-module is a composition series as $X$-module.

Proof. By definition, $\operatorname{Soc}\left(\left.V\right|_{\left[X^{\circ}, X^{\circ}\right]}\right)$ is a completely reducible $\left[X^{\circ}, X^{\circ}\right]$-module, on which $u$ acts as a single Jordan block since that property passes to quotients and submodules. So by Lemma 3.1, it is an irreducible $X$-module. The claim now follows by induction on the length of the socle series of $\left.V\right|_{\left[X^{\circ}, X^{\circ}\right]}$.

Lemma 3.5. Let $N \unlhd H$ be groups with $H=N\langle v\rangle$. Assume that $v$ has order $p^{a}$ and acts by an inner automorphism on $N$. If $N$ does not contain elements of order $p^{a}$ then $H=N \circ\langle z\rangle$ for some element $z \in H$ of infinite order or of order a multiple of $p^{a}$.

Proof. By assumption there is $y \in N$ such that $z:=v y$ centralises $N$. As $z$ centralises $y$, either both $y$ and $z$ are of infinite order, or both have finite order and then the order $p^{a}$ of $v=z y^{-1}$ divides the least common multiple of $|z|$ and $|y|$. In the latter case, $|y|$ is not divisible by $p^{a}$ by assumption, which implies that $p^{a}$ divides $|z|$. Clearly, $\langle N, z\rangle=\langle N, v\rangle=H$, whence our claim.

Lemma 3.6. Let $H \leq \mathrm{SL}(V)$ be a group, and assume that all composition factors of $\left.V\right|_{H}$ are mutually non-isomorphic. Then $Z(H)$ contains no non-trivial unipotent elements.

Proof. Let $u \in Z(H)$ be unipotent. Set $x=u-1 \in C_{\operatorname{End}(V)}(H)$, so $x$ acts as 0 on any irreducible $k H$-subquotient of $V$. Let $V_{1}=\operatorname{ker} x$ and set $\bar{V}=V / V_{1}$. If $x \neq 0$ then $V_{1} \neq V$, so $\bar{V} \neq 0$. Let $\bar{U} \leq \bar{V}$ be an irreducible $k H$-submodule. Then $x . U \leq V_{1}$ where $U$ is the full preimage of $\bar{U}$ in $V$. But then

$$
x . U \cong U /(U \cap \operatorname{ker} x)=U / V_{1}=\bar{U}
$$

is a simple submodule of $V_{1}$ isomorphic to $\bar{U}$, contradicting our assumption. Thus $x=0$ and so $u=1$. 
We now use results of McNinch on semisimplicity of low-dimensional modules in order to study extensions of irreducible modules for simple algebraic groups on which a full Jordan block acts.

Proposition 3.7. Let $X=X^{\circ}\langle u\rangle \leq \mathrm{SL}(V)$ with $X^{\circ}$ a simple algebraic group and $u$ regular unipotent in $\mathrm{SL}(V)$. If $V$ has at most two $X$-composition factors then $X$ acts irreducibly on $V$.

Proof. By [22, Prop. 2.11] we may assume that $X$ is not connected. We argue by contradiction and so assume $V$ has two $X$-composition factors $V_{1}, V_{2}:=V / V_{1}$. Then by Lemma 3.4 these are the socle and the head of $V$ as an $X^{\circ}$-module. Applying Proposition 3.2 to $V_{i}$ and invoking the hypothesis that $X^{\circ}$ is simple, we find that 3.2(1) holds with $r=1$. In particular, for each $i$ we have $V_{i}=V_{i 1} \oplus \cdots \oplus V_{i m_{i}}$ with irreducible $X^{\circ}$-modules $V_{i j}$ where $m_{i}=1$, or $p=2$ and $m_{i} \in\{1,2\}$. Note that for any $j$ there must be a non-trivial extension between $V_{1 j}$ and some $V_{2 l}$.

First assume that $m_{1}=m_{2}=1$, so $V_{i}$ is an irreducible $X^{\circ}$-module for $i=1,2$. Since $X^{\circ}$ is simple, $u^{p}$ must act as an inner automorphism on $X^{\circ}$. We claim that the order of $u^{p}$ in its action on $V$ is bounded above by the order of a unipotent element of $X^{\circ}$. Indeed, if the order of $u^{p}$ is larger than that, then by Lemma 3.5 there is a nontrivial central unipotent element in $X$. On the other hand by Lemma 2.15(a) the two composition factors $V_{1}, V_{2}$ are not isomorphic as $X^{\circ}$-modules. This contradicts Lemma 3.6 .

Now $u$ acts by a single Jordan block on both $X^{\circ}$-composition factors, and both are tensor indecomposable for $X^{\circ}$ by Proposition 2.11. Thus each of these two $X$-modules is either trivial or occurs in Table 1 .

By a result of McNinch [16. Thm 1] the possibilities for non-split extensions between such $X^{\circ}$-modules are very restricted. Namely, he shows that all $X^{\circ}$-modules of dimension below a certain explicit bound are either completely reducible or contained on a short list of exceptions in [16, Tab. 5.1.1]. Based on his dimension bounds one sees that there is no non-trivial extension between a module in Table 1 and the trivial $X^{\circ}$-module with $X$ disconnected.

Hence $V_{1}$ and $V_{2}$ are non-trivial. Then again combining Table 1 with [16, Thm 1] we only arrive at the case that $X$ acts as $A_{2} .2$ on $V_{i}$, with $p=2, \operatorname{dim} V_{i}=8$. Here, $V$ has dimension 16 , so $u$ has order 16 so this case cannot occur by our above considerations.

Next consider the case that $m_{1}=p$. Then we have $p=2, X^{\circ}=A_{l}$ with $l \geq 2$ and $\operatorname{dim} V_{1 j}=l+1$. Then $V_{2}$ cannot be trivial as argued in the preceding case. So if $m_{2}=1$, then $V_{2}$ is an irreducible $A_{l^{-}}$and $A_{l} .2$-module on which $u$ acts with a single Jordan block. By Table 1 this implies that $l=2$ and $\operatorname{dim} V_{2}=8$. Recall that here $\operatorname{dim} V_{1}=6$. A regular unipotent element $u$ of $\mathrm{SL}(V)$ has order 16 . But unipotent elements of $A_{2} .2$ have order at most 8. Arguing as in paragraph 2 using Lemmas 3.5 and 3.6 we again reach a contradiction.

If $m_{2}=2$, then $\operatorname{dim} V_{2 j}=l+1$ as well. Now unipotent elements of $A_{l} .2$ have order less than $4(l+1)=\operatorname{dim} V$. So $u$ has bigger order than any unipotent element of $A_{l} .2$. Arguing as before this shows with Lemma 3.6 that $V_{11} \cong V_{21}$ (after possibly renumbering), and so also $V_{11}^{*}=V_{12} \cong V_{22}$. By untwisting we may assume that $V_{11}$ is the natural module. But there is no non-trivial extension between the natural $A_{l}$-module and itself or its dual. This final contradiction completes the proof. 
The extension question for the exceptional modules showing up in Proposition $3.2(2)$ can be discussed in a similar manner:

Proposition 3.8. Let $X=A_{1}^{p} \cdot\langle u\rangle \leq \mathrm{SL}(V)$ with $p \in\{2,3\}$ and $u$ regular unipotent in $\mathrm{SL}(V)$. If $V$ has at most two $X^{\circ}$-composition factors, one of which is the p-fold tensor product of isomorphic 2-dimensional $A_{1}$-modules, then $V$ is an irreducible $X$-module.

Proof. Assume that $V$ has two $X$-composition factors $V_{1}<V$ and $V_{2}=V / V_{1}$, on one of which $X^{\circ}$ acts non-trivially. By passing to the dual, we may assume that this is $V_{2}$. First consider $p=2$. Then $\operatorname{dim} V_{2}=2^{p}=4$ and $\operatorname{dim} V \geq \operatorname{dim} V_{2}+1=5$, so the regular unipotent element $u \in \mathrm{SL}(V)$ has order at least 8 . But it normalises an $A_{1}^{2}$, so by Lemma 3.5 that $A_{1}^{2}$ is centralised by an element of order 4 . Then Lemma 3.6 shows that the two $X^{\circ}$-composition factors must be isomorphic. As there are no non-trivial self-extensions for $X^{\circ}$ by Lemma 2.15)(a), $V$ is in fact completely reducible as an $X^{\circ}$-module, and hence an irreducible $X$-module by Lemma 3.4, contrary to our assumption.

When $p=3$ then note that there cannot be a non-trivial extension between the $A_{1}^{3}$-module $V_{2}$ and the trivial module, since the zero weight is not subdominant to the highest weight of $V_{2}$. Thus we may assume that $X$ also acts by a non-trivial semisimple group on $V_{1}$. Since the smallest dimension of a faithful $A_{1}^{3}$-module is 6 , $\operatorname{dim} V \geq \operatorname{dim} V_{2}+6 \geq 14$, and so $u \in \mathrm{SL}(V)$ has order at least 27 . We can now argue exactly as in the case $p=2$, with an element of order 9 centralising $X^{\circ}$.

We can now prove the main result of this section, establishing Theorem 1 for the classical algebraic groups $G$ of type $A_{l}, B_{l}$ and $C_{l}$. Recall that it suffices to consider any group isogenous to $G$.

Theorem 3.9. Let $G$ be simple of type $A_{l}, B_{l}$ or $C_{l}$ and $X \leq G$ be a reductive subgroup of the form $X=X^{\circ}\langle u\rangle$ for a regular unipotent element $u$ of $G$ with $\left[X^{\circ}, X^{\circ}\right] \neq 1$. Then $X$ does not lie in any proper parabolic subgroup of $G$.

Proof. As noted in Remark 2.1 we may assume $p>0$. If $p=2$ then we need not consider $G=B_{l}$ as it is isogenous to $C_{l}$. Now embed $X \leq G \leq \mathrm{SL}(V)$ via its natural representation. Then by Lemma 2.12, $u$ has a single Jordan block on $V$. If $X$ lies in some proper parabolic subgroup of $G$, with unipotent radical $U$, then $X \leq N_{\mathrm{SL}(V)}(U)$, which by Borel-Tits lies in a proper parabolic subgroup of $\operatorname{SL}(V)$. Thus it is sufficient to establish the result in the case that $G=\operatorname{SL}(V)$. Here the claim is equivalent to showing that $X$ is irreducible on $V$. For this we may replace $X^{\circ}$ by $\left[X^{\circ}, X^{\circ}\right]$ and therefore assume that $X^{\circ}$ is a non-trivial semisimple group. Furthermore, by Remark 2.2 we may also assume that $u$ is transitive on the set of simple components of $X^{\circ}$ by replacing the latter by one of the $u$-orbits. In particular, any component of $X^{\circ}$ acts non-trivially on any $X$-composition factor of $V$ on which $X^{\circ}$ acts non-trivially.

For a contradiction, assume $X$ is reducible on $V$. By passing to a suitable quotient of $V$, where $u$ still acts by a single Jordan block, we may assume that $X$ has exactly two composition factors on $V$. By Lemma 3.4, any composition series for $X$ is a socle series for $X^{\circ}$, which thus also has two layers.

As $X^{\circ} \neq 1$ there is at least one term in the socle series $0<V_{1}<V$ on which $X^{\circ}$ does not act trivially (which we may assume to be the top layer $V_{2}=V / V_{1}$, by going to the dual if necessary). By Proposition $3.2, X^{\circ}$ acts on $V_{2}$ through $Y_{1} \cdots Y_{r}$ 
with isomorphic semisimple groups $Y_{i}$ and $r>0$. Let $V_{2}=W_{1} \oplus \cdots \oplus W_{r}$ be the corresponding decomposition into $X^{\circ}\left\langle u^{r}\right\rangle$ modules as in Remark 3.3, with $W_{i}$ an irreducible $Y_{i}\left\langle u^{r}\right\rangle$-module and $Y_{i}$ acting trivially on $W_{j}$ for $j \neq i$.

We claim that $X^{\circ}$ also acts non-trivially on $V_{1}$. Assume otherwise, so $\operatorname{dim} V_{1}=$ 1. Now $u$ permutes the semisimple factors $Y_{1}, \ldots, Y_{r}$ transitively, as well as the $W_{1}, \ldots, W_{r}$. For $1 \leq i \leq r$ let $\tilde{W}_{i}$ denote the full preimage of $W_{i}$ in $V$. As $Y_{i}\left\langle u^{r}\right\rangle$ stabilises $W_{i}$, it acts on $\tilde{W}_{i}$. If $Y_{i}$ is simple, then by Proposition 3.7. $u^{r}$ cannot act with a single Jordan block on any of the $\tilde{W}_{i}$. If $Y_{i}$ is not simple, so we are in case (2) of Proposition 3.2, we reach the same conclusion by Proposition 3.8. With $\operatorname{dim} V=r \operatorname{dim} W_{1}+1$ this contradicts the fact that by Lemma 2.3, $u^{r}$ has at least one Jordan block of size $\operatorname{dim} W_{1}+1$ on $V$.

Thus, $X^{\circ}$ acts non-trivially on $V_{1}$ and on $V_{2}$. So we have $V_{s}=W_{s 1} \oplus \cdots \oplus W_{s r}$, where $W_{s i}$ is an irreducible $Y_{i}\left\langle u^{r}\right\rangle$-module, for $s=1,2$ and $i=1, \ldots, r$. Then

$$
\begin{aligned}
\operatorname{Ext}_{X^{\circ}}^{1}\left(V_{1}, V_{2}\right) & =\bigoplus_{i} \operatorname{Ext}_{X^{\circ}}^{1}\left(W_{1 i}, W_{2 i}\right) \oplus \bigoplus_{i \neq j} \operatorname{Ext}_{X^{\circ}}^{1}\left(W_{1 i}, W_{2 j}\right) \\
& =\bigoplus_{i} \operatorname{Ext}_{Y_{i}}^{1}\left(W_{1 i}, W_{2 i}\right) \oplus \bigoplus_{i \neq j} \operatorname{Ext}_{X^{\circ}}^{1}\left(W_{1 i}, W_{2 j}\right)
\end{aligned}
$$

by Lemmas 2.13 and 2.14. Now note that the second sum is zero by comparing highest weights (where we use the fact that if there is a non-trivial extension between two simple modules for a semisimple algebraic group then their weights are comparable, see, e.g., [4, II.2.14]). Thus, $\operatorname{Ext}_{Y_{i}}^{1}\left(W_{1 i}, W_{2 i}\right) \neq 0$ for some, and hence, all $i$. So $\left.V\right|_{Y_{1}}=\tilde{W}_{1} \oplus V^{\prime}$ with $\tilde{W}_{1}$ a non-split extension of $W_{11}$ with $W_{21}$ as a $Y_{1}$-module and $Y_{1}$ acting trivially on $V^{\prime}$. Since $u^{r}$ normalises $Y_{1}$, it stabilises this decomposition of $V$. But $u^{r}$ acts with $r$ Jordan blocks of size $\operatorname{dim} V / r=\operatorname{dim} \tilde{W}_{1}$ on $V$, so it must act by a single Jordan block on $\tilde{W}_{1}$.

If $Y_{i}$ is simple, as in case (1) of Proposition 3.2, there is no such extension $\tilde{W}_{1}$ of the two irreducible $Y_{1}\left\langle u^{r}\right\rangle$-modules $W_{11}, W_{21}$ by Proposition 3.7. giving the desired contradiction. Hence we must be in the case that $Y_{1}=A_{1}^{p}, r=m$, and the action on the $W_{2 i}$ is as in Proposition $3.2(2)$. Again, there is no such non-split extension by Proposition 3.8 .

Thus for Theorem 1, as far as simple groups of classical type are concerned, it remains to consider groups of type $D_{l}$.

\section{The CaSe of $\mathrm{SO}(V)$}

In this section we consider the following situation: $\operatorname{dim} V=2 l$ for $l \geq 4$, and $X \leq \mathrm{SO}(V)$ is a (not necessarily connected) reductive subgroup of the form $X=$ $X^{\circ}\langle u\rangle$, with $X^{\circ}$ not a torus, for a regular unipotent element $u$ of $\mathrm{SO}(V)$. Recall from Lemma 2.12(c) that regular unipotent elements of $\mathrm{SO}(V)$ have two Jordan blocks on $V$, of sizes $2 l-1,1$ if $p \neq 2$, and sizes $2 l-2,2$ when $p=2$. 


\subsection{Almost simple groups containing an element with a large Jordan block.}

Proposition 4.1. Let $p=2$ and $G \leq \mathrm{SL}(V)$ be an almost simple algebraic group acting irreducibly on $V$ and such that some unipotent element of $G$ has a Jordan block of size $\operatorname{dim} V-1$. Then $G=\mathrm{SL}(V)$.

Proof. For $G$ a simple group of exceptional type, the irreducible representations of $G$ whose image contains a unipotent element with a single non-trivial Jordan block are classified in [23, Thm 1.1], and only $G_{2}$ in its 6-dimensional representation comes up. But here no unipotent elements have blocks of size 5. (See e.g. 66.) The only almost simple but not simple group of exceptional type is $G=E_{6} .2$. Here, the regular unipotent elements of $G$ have order 32 , but $G$ has no faithful irreducible representation of dimension less than 54 .

Now assume that $G$ is simple, simply connected, of classical type but not equal to $\mathrm{SL}(V)$ and let $d$ denote the dimension of its natural module. Here we may assume $G$ is not an odd-dimensional orthogonal group as we will consider the isogenous symplectic group. The unipotent elements of $G$ have order less than $2 d$. On the other hand, writing $n=\operatorname{dim} V$, an element with a Jordan block of size $n-1$ has order at least $n-1$, so we get $n<2 d+1$. All irreducible representations of such dimensions are known (see e.g. [12, Thms 4.4 and 5.1]), and we find that either $n=d$, or up to twists one of the following holds:

- $G=A_{1}$ with $d=2$ and $V$ is the tensor product of two 2-dimensional modules, or

- $G=A_{d-1}$ with $4 \leq d \leq 5$ and $V$ is the exterior square of the natural module, or

- $G=C_{d / 2}$ for $d \in\{4,6,8\}$ and $V$ is the spin module, or

- $G=D_{d / 2}$ for $d=8,10$ and $V$ is a spin module.

Looking at the precise order of unipotent elements rules out all cases except spin modules for $C_{2}, C_{3}$ and $D_{4}$. In the latter two cases, the image of the representation lies in the 8-dimensional orthogonal group, where there are no unipotent elements with Jordan blocks of size 7 , and for the case of $G=C_{2}$, both 4-dimensional representations have image in the symplectic group where there are also no unipotent elements with a block of size 3. Finally, if $n=d$, by the above remarks we may assume $V$ is the natural representation of $G(\neq \mathrm{SL}(V))$; we note that $G$ does not have unipotent elements of the required Jordan block sizes on $V$ in characteristic 2 (see [9, Lemma 6.2]).

It remains to consider $G$ almost simple of type $A_{m-1} .2$ or $D_{m} .2=\mathrm{GO}_{2 m}$. Here, unipotent elements have order less than $4 m$, so as before we conclude $n<4 m+1$. Now note that the natural module for $A_{m-1}$ is not invariant under the graph automorphism, nor is its exterior square for $m \neq 4$. For $m>4$ the spin modules for $D_{m}$ do not afford representations of $D_{m} .2$. Again with [12] we arrive at the Lie algebra for $A_{2}$, the exterior square of the natural module for $A_{3}$, and modules of dimension $2 m$. All of these embed $G$ into a general orthogonal group, but the latter does not have unipotent elements of the required Jordan type in its natural representation (see [9, Lemma 6.2]). So none of these leads to examples, completing the proof. 


\subsection{A reduction result.}

Proposition 4.2. Assume $p>0$. Let $X=X^{\circ}\langle u\rangle \leq \mathrm{SO}(V)$ be reductive with $\operatorname{dim} V=2 l \geq 6, X^{\circ} \neq 1$ semisimple, and $u$ regular unipotent in $\mathrm{SO}(V)$. Assume that $X$ does not stabilise any non-zero totally singular subspace of $V$. Then one of the following four mutually exclusive cases occurs:

(1) $X=X^{\circ}$ is irreducible on $V$; more specifically, either $X=\mathrm{SO}(V)$, or $l=4$ and $X=B_{3}$;

(2) $p=2, l$ is even, $X=A_{l-1} .2$ is irreducible on $V$ stabilising a pair of complementary totally singular subspaces interchanged by $u$;

(3) there is an orthogonal decomposition $V=V^{\prime} \perp V^{\prime \prime}$ into $X$-submodules $V^{\prime}, V^{\prime \prime}$, where $\operatorname{dim} V^{\prime \prime}=\operatorname{gcd}(p, 2)$ and $V^{\prime}$ is irreducible and tensor indecomposable for $X$; or

(4) $p=2, X$ stabilises a 1-dimensional non-singular subspace $V_{1}$ of $V, X$ acts as a subgroup of $B_{l-1}$ on $V_{1}^{\perp}$ with $u$ having a single Jordan block on $V_{1}^{\perp} / V_{1}$, and there exists no $X^{\circ}$-complement to $V_{1}$ in $V_{1}^{\perp}$.

Proof. First assume that $V$ is a decomposable $X$-module. Then by the Jordan block shape of $u$ we must have $V=V^{\prime} \oplus V^{\prime \prime}$ with $\operatorname{dim} V^{\prime \prime}=\operatorname{gcd}(2, p)$, and $u$ acts with a single Jordan block on both summands. If $X^{\circ}$ acts non-trivially on $V^{\prime}$ then by Theorem [3.9, $X$ acts irreducibly on $V^{\prime}$. Since $\operatorname{dim} V^{\prime}>\operatorname{dim} V / 2$ then $V^{\prime}$ must be non-degenerate, so we obtain an $X$-invariant decomposition $V=V^{\prime} \perp\left(V^{\prime}\right)^{\perp}$. Lemma 2.4 shows that $V^{\prime}$ is tensor indecomposable for $X$, so we reach conclusion (3). On the other hand, if $X^{\circ}$ is trivial on $V^{\prime}$ then it must act faithfully on $V^{\prime \prime}$ and hence $X^{\circ}=A_{1}$ and $p=2$. In particular, $u$ acts by an inner automorphism on $X^{\circ}$ and thus $X^{\circ}$ contains a regular unipotent element of $\mathrm{SO}(V)$ by Lemma 2.9. which is impossible as these have order at least 4 .

So now assume that $V$ is an indecomposable $X$-module. In particular, there is no $X$-invariant non-degenerate non-trivial proper subspace of $V$. Thus, if $V_{1}$ denotes a non-zero $X$-invariant subspace of $V$ of minimal dimension, then either $V_{1}=V$, that is, $X$ acts irreducibly on $V$, or $V_{1}$ is non-singular of dimension 1 and $p=2$. In the latter case $X$ is contained in the stabiliser of $V_{1}$, isomorphic to $B_{l-1}$, and $V_{1}^{\perp}$ is the natural module for $B_{l-1}$. Let's first discuss this situation. Now $u$ is regular unipotent in $B_{l-1}$ by Lemma 2.10, so it has a single Jordan block on $V_{1}^{\perp} / V_{1}$. By Theorem 3.9. $X$ acts irreducibly on $V_{1}^{\perp} / V_{1}$, and thus $\left.\left(V_{1}^{\perp} / V_{1}\right)\right|_{X^{\circ}}$ is a direct sum of non-isomorphic irreducible $X^{\circ}$-modules by Lemma 2.5, all non-trivial as $X^{\circ}$ acts non-trivially on $V_{1}^{\perp} / V_{1}$. Assume that $\left.V_{1}^{\perp}\right|_{X^{\circ}}=V_{1} \oplus N$. As $N \cong V_{1}^{\perp} / V_{1}$ has no trivial $X^{\circ}$-composition factor, this decomposition is $X$-invariant. By dimension reasons, the irreducible $X$-module $N$ must be non-degenerate, but this was excluded before. Thus $V_{1}$ has no $X^{\circ}$-complement in $V_{1}^{\perp}$, and hence (4) holds.

Thus we are left to consider the case that $X$ acts irreducibly on $V$. By Lemma 2.5 then $\left.V\right|_{X^{\circ}}=V_{1} \oplus \cdots \oplus V_{m}$ is a direct sum of non-isomorphic irreducible $X^{\circ}$-modules transitively permuted by $u$. Then $2 l=m r$ with $r=\operatorname{dim} V_{1}>1$ and $m$ is a power of $p$. First assume $u$ has Jordan blocks of sizes $2 l-1,1$ on $V$. Induction from Lemma 2.3 shows that $u^{m}$ has $m-1$ Jordan blocks of size $r$, one Jordan block of size $r-1$ and one Jordan block of size 1 on $V$. Since $u^{m}$ has the same Jordan blocks on each $V_{i}$ by transitivity, the only compatible solution is $m=1$. If $u$ has Jordan blocks of sizes $2 l-2,2$ on $V$ (and so in particular $p=2$ ) then Lemma 2.3 shows that either $m=1$, or $u^{m}$ has $m-2$ blocks of size $r$, and 2 blocks of sizes 
$r-1,1$ each. This forces $m=2$. In conclusion either $m=1$, or $m=p=2$. Let us consider these two cases in turn.

If $m=1$, that is, if $\left.V\right|_{X^{\circ}}$ is irreducible, then $X^{\circ}$ is simple by Lemma 2.7 as none of (1)-(3) there can occur here. If $X^{\circ}=X=\mathrm{SO}(V)$ we are in case (1) of our statement. If not, then by Proposition 2.11 the only possibility for $l \geq 4$ is again the one given in (1). For $l=3$, using that $X^{\circ}$ must also be irreducible on the natural 4-dimensional module $U$ for $A_{3} \cong D_{3}$ (e.g. by Borel-Tits), Proposition 2.11 implies that we must have $X^{\circ}=A_{1}$ with $p \geq 5$ or $X^{\circ}=C_{2}=B_{2}$. But in neither of these cases does $X^{\circ}$ act irreducibly on the exterior square $\Lambda^{2}(U)=V$, so this case does not occur here.

Now consider the case where $m=p=2$ so that the Jordan blocks of $u^{2}$ on $V_{i}$, $i=1,2$, have sizes $l-1,1$. We claim that $V_{1}$ is totally singular. For otherwise, $V_{1}$ is non-degenerate and thus so is $V_{1}^{\perp}$ which must be $V_{2}$, and therefore $X$ is contained in the stabiliser in $\mathrm{SO}(V)$ of the orthogonal decomposition $V=V_{1} \perp V_{2}$, hence in $\mathrm{GO}\left(V_{1}\right) \mathrm{GO}\left(V_{2}\right) \cdot 2 \cap \mathrm{SO}(V)$. But by [17, Thm $\left.\mathrm{B}(\mathrm{ii})(\mathrm{a})\right]$ there is no reductive maximal subgroup of $\mathrm{SO}(V)$ containing this stabiliser and a regular unipotent element of $\mathrm{SO}(V)$.

We thus have that $V_{1}$ is totally singular as claimed; then so is its image $V_{2}$ under $u$. Hence $X$ stabilises a decomposition of $V$ into a direct sum of maximal totally singular subspaces and thus $X \leq \mathrm{GL}_{l} .2$. According to Lemma 2.12)(d) when $l$ is odd, the regular unipotent elements of the stabiliser $\mathrm{GL}_{l} .2$ of such a decomposition have a single Jordan block, so cannot lie in $\mathrm{SO}(V)$. Thus $X \leq \mathrm{GL}_{l} \cdot 2 \cap \mathrm{SO}(V)=$ $\mathrm{GL}_{l}$, which does not contain elements with a Jordan block of size $2 l-2$. On the other hand, when $l$ is even the stabiliser $\mathrm{GL}_{l} .2$ contains regular unipotent elements of $\mathrm{SO}(V)$. Lemma 2.7 now implies that $X^{\circ}$ is simple, since the case (3) with $\operatorname{dim} V_{1}=9$ cannot occur here as $l$ is even. By Proposition 4.1 this gives the examples in (2).

In what follows we investigate further the case (4) of the preceding result.

Proposition 4.3. In the situation of Proposition 4.2(4), the following hold:

(a) $X^{\circ}=Y_{1} \cdots Y_{r}$, with pairwise isomorphic factors $Y_{i}=B_{n}, Y_{i}=C_{n}$ (with $n \geq 1)$ or $Y_{i}=G_{2}$, permuted transitively by $u$; and

(b) there is a decomposition $V_{1}^{\perp} / V_{1} \cong \bigoplus_{i=1}^{r} U_{i}$ into $Y_{i}\left\langle u^{r}\right\rangle$-modules $U_{i}$, irreducible for $Y_{i}$ and transitively permuted by $u$, and on which $u^{r}$ acts by a single Jordan block, with $\operatorname{dim} U_{i}=2 n$ when $Y_{i}=B_{n}$ or $C_{n}$, respectively $\operatorname{dim} U_{i}=6$ when $Y_{i}=G_{2}$.

Proof. We keep the notation from Proposition 4.2(4). Recall that here $p=2$. As $u$ acts by a single Jordan block on $V_{1}^{\perp} / V_{1}$, this is an irreducible $X$-module by Theorem 3.9. So by Proposition 3.2 there is a decomposition $X^{\circ}=Y_{1} \cdots Y_{r}$ with $u$ transitively permuting the semisimple factors $Y_{i}$.

We first show that we are not in case (2) of Proposition 3.2. Suppose the contrary. Then $Y_{i}=X_{2 i-1} X_{2 i}$ with $X_{j}=A_{1}, r=m$, and $V_{1}^{\perp} / V_{1}=U_{1} \oplus \cdots \oplus U_{r}$, where $U_{i}$ is an $X_{2 i-1} X_{2 i}$-module which is a twist of a tensor product of two natural modules for $A_{1}$ and the $U_{i}$ are transitively permuted by $u$. Let $\tilde{U}_{i}$ denote the full preimage of $U_{i}$ in $V_{1}^{\perp}$, so $V_{1}^{\perp}=\sum \tilde{U}_{i}$. The Künneth formula [20, Lemma 3.3.6] shows that $\operatorname{Ext}_{X_{2 i-1} X_{2 i}}^{1}\left(U_{i}, k\right)=0$, whence $\tilde{U}_{1}=V_{1} \oplus N_{1}$, with $N_{1} \cong U_{1}$. So there is a similar decomposition for all of the $\tilde{U}_{i}$, leading to a decomposition of the $X^{\circ}$-module $V_{1}^{\perp}=V_{1} \oplus\left(\sum N_{i}\right)$, contradicting Proposition 4.2(4). 
Hence we are in case (1) of Proposition 3.2 and all $Y_{i}$ are simple. Let us write $\bar{i}:=i(\bmod r)$. Now $V_{1}^{\perp} / V_{1}=U_{1} \oplus \cdots \oplus U_{m}$, where the $U_{i}$ are irreducible tensor indecomposable $Y_{\bar{i}}$-modules, transitively permuted by $u$, and $u^{m}$ acts by a single Jordan block on each. Hence the possibilities for $\left(Y_{\bar{i}}\left\langle u^{m}\right\rangle, U_{i}\right)$ are as listed in Table1, Moreover, arguing as in the preceding paragraph we see that $\operatorname{Ext}_{Y_{\bar{i}}}^{1}\left(U_{i}, k\right) \neq 0$. Now by [16] all pairs $\left(Y_{\bar{i}}\left\langle u^{m}\right\rangle, U_{i}\right)$ have $\operatorname{Ext}_{Y_{\bar{i}}}^{1}\left(U_{i}, k\right)=0$ except possibly for $Y_{\bar{i}}=B_{n}$, $Y_{\bar{i}}=C_{n}$ or $Y_{\bar{i}}=G_{2}$ with $\operatorname{dim} U_{i}$ as claimed, so by Proposition 3.2 we have $r=m$ and we get (a) and (b).

Proposition 4.4. Let $X=X^{\circ}\langle u\rangle \leq \mathrm{SO}(V)$ be reductive with $\operatorname{dim} V=2 l \geq 8$, $X^{\circ} \neq 1$ semisimple, and $u$ regular unipotent in $\mathrm{SO}(V)$. Then $X$ does not stabilise a maximal totally singular subspace of $V$.

Proof. Arguing by contradiction, assume that $X$ stabilises some maximal totally singular $W<V$. Then its stabilizer $P$ has Levi factor $L=\mathrm{GL}(W)$. By Lemma 2.8, for $Q=R_{u}(P)$ the image of $X$ in $P / Q \cong L$ contains a regular unipotent element of $L$, and $X$ acts irreducibly on $W$ by Theorem 3.9 and hence on its dual $V / W$. Note that $X^{\circ}$ acts non-trivially on $W$.

Let us first consider the case when $p=2$. Assume that $u$ acts with different orders on $W$ and on $V$. Then a non-trivial power $v$ of $u$, which we may take to be an involution, is in the kernel of the representation on $W$. Letting $\varphi_{1}: P \rightarrow \operatorname{GL}(W)$ denote the projection into the Levi factor of $P$, we thus have $\left[v, X^{\circ}\right]$ lies in the normal subgroup $X^{\circ}$, and has image $\varphi_{1}\left(\left[v, X^{\circ}\right]\right)=\left[\varphi_{1}(v), \varphi_{1}\left(X^{\circ}\right)\right]=1$, so in fact $\left[v, X^{\circ}\right]=1$, that is, $v$ centralises $X^{\circ}$. As $u^{2}$ has two Jordan blocks $J_{1}$, the element $v$ has Jordan blocks $J_{2}^{a} \oplus J_{1}^{b}$ with $a \geq 1, b \geq 2$ on $V$. (Here $J_{i}$ denotes a Jordan block of size $i$.) Then by [9, Thm 3.1], setting $C=C_{\mathrm{SL}(V)}(v)$, we have that $C / R_{u}(C) \cong \mathrm{GL}_{a} \mathrm{GL}_{b}$ and the action of this group on $V$ is via two copies of the natural module for $\mathrm{GL}_{a}$ and one copy of the natural module for $\mathrm{GL}_{b}$. As $X \subseteq C$, the dimensions of the $X$-composition factors on $V$ (two factors of dimension $l$ ) must be obtained as a refinement of the dimensions $a, a, b$ coming from the action of $\mathrm{GL}_{a} \mathrm{GL}_{b}$, which is not possible. So $u$ has the same order on $W$ as on $V$. As $u$ has a Jordan block of size $l$ on $W$, and blocks of sizes $2 l-2,2$ on $V$, we must have $l=2^{f}+1 \geq 5$. So we have proved that $l$ is odd when $p=2$.

Now let $p$ be arbitrary again and write $\left.W\right|_{X^{\circ}}=W_{1} \oplus \cdots \oplus W_{r}$ for the decomposition of $W$ into pairwise non-isomorphic irreducible $X^{\circ}\left\langle u^{r}\right\rangle$-modules as in Remark 3.3, with $u$ transitively permuting the summands. Note that since $l=\operatorname{dim} W$ has to be odd when $p=2$ we cannot be in case (1) of Proposition 3.2. As $W$ and $V / W$ are dual to each other, then $\left.(V / W)\right|_{X^{\circ}} \cong W_{1}^{*} \oplus \cdots \oplus W_{r}^{*}$ with $W_{i}^{*}$ dual to $W_{i}$. Let $V_{1}$ be the $X^{\circ}\left\langle u^{r}\right\rangle$-submodule of $V$ with composition factors $W_{1}$ and $W_{1}^{*}$ (if $V$ is not completely reducible as an $X^{\circ}\left\langle u^{r}\right\rangle$-module, this exists by Lemma 2.13 together with Remark 3.3). Then the transitive action of $u$ yields $\left.V\right|_{X^{\circ}}=\bigoplus_{i=1}^{r} u^{i-1}\left(V_{1}\right)$. From the block structure of $u$, as in the proof of Proposition 4.2 this implies that $r=1$ or $r=p=2$. But the latter cannot occur as $l$ is odd when $p=2$. So $r=1$, and $V_{1}=V$ is an extension of an irreducible $X^{\circ}$-module by its dual, on both of which $u^{r}=u$ has a single Jordan block. Assume that this extension splits. If $\left.\left.W\right|_{X^{\circ}} \cong W^{*}\right|_{X}$ 。 is self-dual, so $\left.V\right|_{X} \circ$ is homogeneous, then it is an irreducible $X$-module by Lemma 2.6, a contradiction. Else, the decomposition $V=W \oplus W^{*}$ is $X$-invariant, contrary to the block structure of $u$. 
So $\left.V\right|_{X}$ is a non-trivial extension, and since by Lemma 2.15)(a) there are no selfextensions for simple groups, $W$ cannot be a self-dual $X^{\circ}$-module. Moreover, still by Proposition 3.2, $X^{\circ}$ is now either simple or $X^{\circ}=A_{1}^{p}$ with $p \leq 3$. The second possibility is ruled out as $W$ is not self-dual. The possible non-self-dual $W$ for $X^{\circ}$ simple are listed in Table 1, and we find that $X^{\circ}=A_{l}$ with $l \geq 2$. But according to [16. Cor. 1.1.1], there is no non-trivial extension of a twist of the natural module of $A_{l}$ with its dual.

The following proposition treats the special case arising out of Proposition 4.3 when $r=1$. By our Lemma 2.9, $X^{\circ}$ contains a regular unipotent element. This case should have been treated in [22] but the argument there is incomplete in precisely this setting. So we have included a proof here.

Proposition 4.5. Let $X$ be a simple algebraic group with $X<G=\mathrm{SO}(V)$, $\operatorname{dim} V=2 l \geq 8$, defined over a field of characteristic 2 . Assume that $X$ stabilises a non-zero totally singular subspace of $V$. Then $X$ does not contain a regular unipotent element of $G$.

Proof. Assume $u \in X$ is regular unipotent. Choose a maximal totally singular $X$-invariant subspace $W$. Then the stabilizer $P=N_{G}(W)$ has Levi factor $L=$ $\mathrm{GL}(W) \mathrm{SO}\left(W^{\perp} / W\right)$ and the projection of $X$ into the second factor does not lie in a proper parabolic subgroup.

By Lemma 2.8, for $Q=R_{u}(P)$ the image of $X$ in $P / Q \cong L$ contains a regular unipotent element of $L$. Thus, if $\operatorname{dim} W>1$, the projection onto the factor $\operatorname{GL}(W)$ of $L$ is injective when restricted to $X$. But the order of a regular unipotent element in $\operatorname{GL}(W)$ is strictly less than the order of $u$ unless possibly when $W$ is maximal totally singular, and this latter case does not occur by Proposition 4.4. Hence we need only consider the case where $\operatorname{dim} W=1$. Let $\pi: P \rightarrow \mathrm{SO}\left(W^{\perp} / W\right)$ be the natural projection. Since $\pi(X)$ does not lie in a proper parabolic subgroup of $\mathrm{SO}\left(W^{\perp} / W\right)$, by [10, Lemma 2.2], one of:

(i) $\left.\left(W^{\perp} / W\right)\right|_{\pi(X)}=W_{1} \perp W_{2} \perp \cdots \perp W_{t}$, with all $W_{i}$ non-degenerate, inequivalent and irreducible $\pi(X)$-modules; or

(ii) $\pi(X)$ stabilizes a nonsingular 1-space of $W^{\perp} / W$.

In the first case, when $t=1$, since $\pi(X)$ contains a regular unipotent element of $\mathrm{SO}\left(W^{\perp} / W\right)$, Proposition 2.11 implies that either $\operatorname{dim}\left(W^{\perp} / W\right)=8, X=B_{3}$ and the action of $X$ on $W^{\perp} / W$ is via a spin module, or $X=\mathrm{SO}\left(W^{\perp} / W\right)$. In both cases, there are no non-trivial extensions between $W^{\perp} / W$ and the trivial $X$-module and we deduce that $X$ (and hence $u$ ) lies in the Levi factor, a contradiction.

In the first case, with $t \geq 2$, the Jordan block structure of $u$ then implies that $t=2$ and we may assume $\operatorname{dim} W_{2}=2$. But then the projection of $\pi(X)$ to $\mathrm{SO}\left(W_{2}\right)$ is trivial as the latter is a torus, contradicting the Jordan block structure of $\pi(u)$.

So we now have that $\pi(X)$ lies in the stabilizer of a nonsingular 1-space of $W^{\perp} / W$; let $U / W$ be such a subspace, so that $X$ stabilizes the flag $0<W<U<$ $U^{\perp}<W^{\perp}<V$.

Now Proposition 4.2 (4) gives that $\pi(u)$ has one block on $U^{\perp} / U$ and so by Table1 we are left with the following irreducible actions on $U^{\perp} / U$ :

- $\pi(X)=C_{l-2}$ or $B_{l-2}$;

- $X=G_{2}, l=5$.

In the first case, we deduce that $\pi(X)=B_{l-2}$ (the full stabilizer in $\mathrm{SO}\left(W^{\perp} / W\right.$ ) of a non-singular 1 -space), and so $W^{\perp} / W$ is a $(2 l-2)$-dimensional tilting module 
for $\pi(X)$. In particular, there is no extension of this module by a trivial and we find that $X$ lies in a Levi factor of $G$. This rules out the case where $X=B_{l-2}$ or $C_{l-2}$.

In the second case, we have $G_{2} Q / Q \leq B_{3} Q / Q \leq D_{4} T_{1} \cong P / Q$, and the action of $X$ on $W^{\perp} / W$ is as an 8-dimensional indecomposable tilting module (see [8, Lemma 9.1.1]) and as above there is no non-trivial extension with the trivial module. So $X$ lies in a proper Levi factor of $G$, and hence cannot contain a regular unipotent element. This final contradiction completes the proof.

Proposition 4.6. Let $X=X^{\circ}\langle u\rangle \leq \mathrm{SO}(V)$ be reductive with $\operatorname{dim} V=2 l \geq 8$, $X^{\circ} \neq 1$ semisimple, and $u$ regular unipotent in $\mathrm{SO}(V)$. Assume that $X$ lies in a proper parabolic subgroup of $\mathrm{SO}(V)$. Then, with $W$ an $X$-invariant totally singular subspace of $V$ of maximal possible dimension, we have: $0<W<W^{\perp}$, $\operatorname{dim} W^{\perp} / W \geq 6$, and $X^{\circ}$ acts non-trivially on $W^{\perp} / W$ and is not completely reducible on $W^{\perp}$.

Proof. Let $0<W<V$ be as in the statement. So $X$ lies in a proper parabolic subgroup of $\mathrm{SO}(V)$ with Levi complement $\mathrm{GL}(W) \mathrm{SO}\left(W^{\perp} / W\right)$. By Lemma 2.8 the image of $u$ in the Levi factor is again regular unipotent, in particular $u$ acts by a single Jordan block on $W$. Also note that $X^{\circ}$ acts non-trivially on $W^{\perp}$, as otherwise it would act trivially on $V / W^{\perp} \cong W^{*}$ as well and hence on all of $V$. By Proposition 4.4. $W$ cannot be maximal totally singular.

So, we now assume that $0<W<W^{\perp}$, and thus $X$ is reducible on $W^{\perp}$. By Theorem 3.9 this implies that $u$ cannot have a single Jordan block on $W^{\perp}$. Thus, $u$ has exactly two Jordan blocks on $W^{\perp}$, one of which has size at $\operatorname{most} \operatorname{gcd}(p, 2)$. We set $n:=\operatorname{dim} W^{\perp}$.

Note that $X$ does not stabilise any non-zero totally singular subspace of $W^{\perp} / W$ by the choice of $W$. This then implies that $X^{\circ}$ acts non-trivially on $W^{\perp} / W$ since $u$, lying in a Borel subgroup, has some totally singular fixed points. In particular $\operatorname{dim} W^{\perp} / W \geq 4$. In fact, if $\operatorname{dim}\left(W^{\perp} / W\right)=4$ then the image of $X^{\circ}$ in $\mathrm{SO}_{4}$ and hence $X^{\circ}$ itself is either $A_{1}$ or $A_{1}^{2}$. If $X^{\circ}=A_{1}$ then $u$ acts on it by an inner automorphism, so $X^{\circ}$ contains a regular unipotent element of $\mathrm{SO}(V)$ by Lemma 2.9. contradicting the main result of [22]. Similarly, if $X^{\circ}=A_{1}^{2} \cong \mathrm{SO}_{4}$ is the full Levi factor and thus $X=X^{\circ}$, we conclude by the same argument. Hence we have $\operatorname{dim} W^{\perp} / W \geq 6$. If $X^{\circ}$ is not completely reducible on $W^{\perp}$, then we arrive at the conclusion.

So now assume that $W^{\perp}$ is a completely reducible $X^{\circ}$-module. We will show that this leads to a contradiction. Write $\left.W^{\perp}\right|_{X^{\circ}}=V_{1} \oplus \cdots \oplus V_{m}$ for the decomposition of $W^{\perp}$ into its $X^{\circ}$-homogeneous components. Then $u$ permutes these components and can have at most two orbits on $\left\{V_{1}, \ldots, V_{m}\right\}$, as it has two Jordan blocks on $W^{\perp}$.

Case 1. We first discuss the case where $u$ is transitive on $\left\{V_{1}, \ldots, V_{m}\right\}$. Arguing precisely as in the proof of Proposition 4.2 the Jordan block shape of $u$ forces either $m=1$, or $m=p=2$. We consider these two cases in turn.

If $m=1$, that is, if $\left.W^{\perp}\right|_{X}$ 。 is homogeneous, then by Lemma 2.6] we find that $\operatorname{dim} W^{\perp}=4$, as $X^{\circ}$ is reducible on $W^{\perp}$, contradicting $\operatorname{dim} W^{\perp} / W \geq 6$.

If $m=p=2$, one checks that the Jordan blocks of $u^{2}$ on $V_{i}, i=1,2$, have sizes $n / 2-1,1$. As $V_{i}$ is $X^{\circ}\left\langle u^{2}\right\rangle$-invariant and homogeneous as an $X^{\circ}$-module, it 
must be irreducible for $X^{\circ}$ by Lemma 2.6. Now $u$ interchanges $V_{1}$ and $V_{2}$, so $W^{\perp}$ is irreducible for $X$, a contradiction.

Case 2. So now assume that $u$ has two orbits on $\left\{V_{1}, \ldots, V_{m}\right\}$. Let $V^{\prime}, V^{\prime \prime}$ denote the subspaces spanned by these orbits, with $\operatorname{dim} V^{\prime \prime}=\operatorname{gcd}(2, p)$. Then $u$ acts with a single Jordan block on each of them.

Consider first the case that $X^{\circ}$ acts non-trivially on $V^{\prime}$. Then, since $u$ has a single Jordan block on $V^{\prime}$ we obtain by Theorem 3.9 that $V^{\prime}$ is an irreducible $X$-module. Then either $V^{\prime}=W$ or $W \cap V^{\prime}=0$. In the first case $W^{\perp} / W$ must be isomorphic to $V^{\prime \prime}$ (as $W \neq W^{\perp}$ ), contradicting that $\operatorname{dim} W^{\perp} / W \geq 6$. If on the other hand $W \cap V^{\prime}=0$ then $V^{\prime}$ is isomorphic to a submodule of $W^{\perp} / W$. Thus the orthogonal group $\mathrm{SO}\left(W^{\perp} / W\right)$ of dimension $n-\operatorname{dim} W$ contains a unipotent element with a Jordan block of size at least $n-\operatorname{gcd}(2, p)$. By the knowledge of possible Jordan block shapes (see [9, Lemma 6.2]) this is not possible as $\operatorname{dim} W \geq 1$.

Finally, in Case 2 it remains to discuss the situation where $X^{\circ}$ acts trivially on $V^{\prime}$. Then it must act irreducibly and faithfully on $V^{\prime \prime}$ and hence $X^{\circ}=A_{1}$ and $p=2$. In particular, $u$ acts by an inner automorphism on $X^{\circ}$ and thus $X^{\circ}$ contains a regular unipotent element of $\mathrm{SO}(V)$ by Lemma 2.9. which is impossible by order considerations.

\subsection{Proof of Theorem 1 for $\mathrm{SO}(V), \operatorname{dim} V=2 l$.}

Theorem 4.7. Let $X=X^{\circ}\langle u\rangle \leq G:=\mathrm{SO}(V)$, where $\operatorname{dim} V=2 l \geq 8$, be a reductive subgroup, with $u$ a regular unipotent element of $G$. Assume that $\left[X^{\circ}, X^{\circ}\right] \neq 1$. Then $X$ does not lie in any proper parabolic subgroup of $G$.

Proof. It suffices to prove the claim for $\left[X^{\circ}, X^{\circ}\right]\langle u\rangle$, and hence we may and will assume that $X^{\circ}=\left[X^{\circ}, X^{\circ}\right]$ is semisimple. Moreover, by Remark 2.2 we may assume that $X^{\circ}$ is the product over a single $u$-orbit of simple components. Assume that $X$ lies in a proper parabolic subgroup $P$ of $G=\mathrm{SO}(V)$. Then there is an $X$-invariant flag $0<W \leq W^{\perp}<V$ with $W$ totally singular and dual to $V / W^{\perp}$ as an $X$-module, and $W^{\perp} / W$ non-degenerate. We choose $P$ such that $\operatorname{dim} W$ is maximal possible (and so $\operatorname{dim} W^{\perp} / W$ is minimal). Hence, we are in the setting of Proposition 4.6.

By the choice of $W$ and since $\operatorname{dim} W^{\perp} / W \geq 6$, we have that $X$ acts as in (1)-(4) of Proposition 4.2 on $W^{\perp} / W$. Also, by Lemma 2.8, $u$ acts with a single Jordan block on $W$ as well as on $V / W^{\perp}$, and with two Jordan blocks on $W^{\perp} / W$.

Case 1. First assume that $X^{\circ}$ acts non-trivially on $W$ and thus that $\operatorname{dim} W \geq 2$. By Proposition 4.6. $X^{\circ}$ acts non-trivially on $W^{\perp} / W$ as well, which has dimension at least 6 .

Case 1a. We first discuss the case where $p=2$. Let $\varphi_{i}, i=1,2$, be the projections of $P$ into the two factors of the Levi subgroup $\mathrm{GL}(W), \mathrm{SO}\left(W^{\perp} / W\right)$ respectively. Since $\varphi_{1}(X)$ is irreducible on $W$ by Theorem 3.9. it cannot lie in a proper parabolic subgroup of GL $(W)$, and by the choice of $W$, neither is $\varphi_{2}(X)$ contained in a proper parabolic subgroup of $\mathrm{SO}\left(W^{\perp} / W\right)$. Write $m=\operatorname{dim} W$ and so $\operatorname{dim} W^{\perp} / W=$ $2(l-m)$. We know that $\varphi_{1}(u)$ is a single Jordan block, and $\varphi_{2}(u)$ has a block of size $2(l-m)-2$ and one of size 2 , by Lemma 2.8. Since $m<l$ by assumption, $\varphi_{1}(u)$ has order smaller than $|u|$, so some power $u^{s} \neq 1$ with $s>1$ lies in $\operatorname{ker} \varphi_{1}$; we choose $s$ minimal with this property. As before, we see that $u^{s}$ must centralise $X^{\circ}$. But then $\varphi_{2}\left(u^{s}\right)=1$ as well, as otherwise $\varphi_{2}\left(u^{s}\right)$ is a non-trivial unipotent element 
of $\mathrm{SO}\left(W^{\perp} / W\right)$ centralised by $\varphi_{2}(X)$, whence by Borel-Tits, $\varphi_{2}(X)$ lies in a proper parabolic subgroup of $\mathrm{SO}\left(W^{\perp} / W\right)$, which is not the case. Note that no smaller power of $u$ lies in $\operatorname{ker} \varphi_{2}$ as otherwise that element would (as before) centralise $X^{\circ}$, forcing $\varphi_{1}(X)$ to lie in a proper parabolic of $\mathrm{GL}(W)$, again a contradiction. So $\varphi_{1}(u), \varphi_{2}(u)$ have the same order $s$.

Recall that $u$ has a single block of size $m$ on $W$ and blocks of sizes $2 l-2 m-2$ and 2 on $W^{\perp} / W$. Also, $u$ has two blocks on $W^{\perp}$, one of size 1 or 2 . But the first possibility is ruled out as $(u-1)^{2 l-2 m-2} W^{\perp} \subseteq W$ and so $(u-1)^{m}(u-1)^{2 l-2 m-2} W^{\perp}=0$.

We now show that $u$ has order $2 s$ on $W^{\perp}$, that is, order twice as large as its order on $W$ (and on $W^{\perp} / W$ ). Let $f$ be minimal such that $2^{f} \geq m$, so $f$ is also minimal so that $2^{f} \geq 2 l-2 m-2$. Hence we have $2 m>2^{f} \geq m$ and $4 l-4 m-4>2^{f} \geq 2 l-2 m-2$. In particular, $2^{f+1} \geq 2 l-m-2$. Since also $2^{f}=2^{f-1}+2^{f-1}<m+2 l-2 m-2=2 l-m-2$, the order of $u$ in its action on $W^{\perp}$ is $2 s$. In particular, $u^{s}$ acts as an involution $J_{2}^{a} \oplus J_{1}^{b}$ on $W^{\perp}$, with $a \geq 1$ and $b \geq 2$ (as $s>1$ ). As in a previous proof, the centraliser of $u^{s}$ in $\operatorname{SL}\left(W^{\perp}\right)$ has composition factors of dimensions $a, a, b$ on $V$, and as $u^{s}$ centralises $X$, the $X$-composition factors on $W^{\perp}$ must be obtained as a refinement of this.

Note that since $a \geq 1$ and $b \geq 2$, there are at least three composition factors and so $X$ cannot act irreducibly on $W^{\perp} / W$, ruling out configurations (1) and (2) of Proposition 4.2. Considering the cases in (3) and (4), we see that $X$ has composition factor dimensions on $W^{\perp}$ among

$$
\{m, 2 l-2 m-2,2\} \text { and }\{m, 2 l-2 m-2,1,1\} .
$$

As $l \geq 8$ and $2 l-2 m \geq 6$, the first case yields $a=m=2 l-2 m-2$ and $b=2$. In the second case, the natural module for $\mathrm{GL}_{a}$ must remain irreducible for $X$, as else there are five composition factors, and so either $a=1$, or $a=m=2 l-2 m-2$ and $b=2$.

If $a=1$, then $u^{s}$ has one block of size 2 and the remaining blocks of size 1 on $W^{\perp}$. By Lemma 2.3 this can only happen if $2 l-m-2=2^{c}+1$ for some $c$, so $u$ has order $2^{c+1}$ on $W^{\perp}$ and by the previous analysis, its order on $W$ and on $W^{\perp} / W$ is $2^{c}$ (so $c=f$ as above). But this implies $2^{c-1}<m<2^{c-1}+1$, a contradiction.

Hence we have $b=2$ and $a=m=2 l-2 m-2$ and $u^{s}$ has exactly two blocks of size 1 on $W^{\perp}$ (coming from the block $J_{2}$ of $u$ on $W^{\perp}$ ), while the one block of size $2 l-m-2$ produces only blocks of size 2 for $u^{s}$. So $2 l-m-2$ is a power of 2 , say $2 l-m-2=2^{c}$. Thus, $u$ has order $2^{c}$ on $W^{\perp}$ and the order of $u$ on $W$ is $2^{c-1}$, so $2^{c-1} \geq m$ and $2^{c-1} \geq 2 l-2 m-2$, forcing $m=2^{c-1}=2 l-2 m-2$.

We claim that neither of the possible actions of $X$ on $W^{\perp} / W$ (as in Proposition $4.2(3)$ and (4)) is consistent with this. First suppose we have the configuration of Proposition 4.2(3), where $W^{\perp} / W=V_{1} \oplus V_{2}, X$ is irreducible on $V_{1}$ and $\operatorname{dim} V_{2}=2$. Let $\tilde{V}_{i}$ be the $X$-submodules of $W^{\perp}$ such that $\tilde{V}_{i} / W=V_{i}, i=1,2$. On each of these $X$ acts reducibly and so $u$ has at least two Jordan blocks. Hence we have $\operatorname{dim} \tilde{V}_{i}^{u} \geq 2$ and $\tilde{V}_{1} \cap \tilde{V}_{2}=W$, whence $\operatorname{dim}\left(\tilde{V}_{1}^{u} \cap \tilde{V}_{2}^{u}\right)=\operatorname{dim} W^{u}=1$ as $u$ has a single Jordan block on $W$, which gives $\operatorname{dim}\left(\tilde{V}_{1}+\tilde{V}_{2}\right)^{u} \geq 3$, contradiction.

So finally we are left to consider the case where $X$ acts on $W^{\perp} / W$ as in Proposition 4.2(4). Here we have $0 \leq W \leq V_{1} \leq V_{1}^{\perp} \leq W^{\perp}$, with $\operatorname{dim} W=m$, $\operatorname{dim} V_{1} / W=1=\operatorname{dim} W^{\perp} / V_{1}^{\perp}$ and $\operatorname{dim} V_{1}^{\perp} / V_{1}=m$. Recall that $u$ has order $2 m=2^{c}$ on $W^{\perp}$ and thus the same order on the codimension 1 subspace $V_{1}^{\perp}$, which is again twice the order of $u$ on $W$ and on $W^{\perp} / W$. So again $u^{s}$ acts as an 
involution on $V_{1}^{\perp}$ and the $X$-composition factors are of dimensions $m, 1, m$. This is only consistent with the $\mathrm{GL}_{a} \mathrm{GL}_{b}$ analysis if $b=1$. This is the final contradiction settling Case 1a.

Case 1b. So now we have $p>2$. As $\operatorname{dim}\left(W^{\perp} / W\right) \geq 6$, we may apply Proposition 4.2 to the action of $X$ on $W^{\perp} / W$, and as $p>2$ we are in either case (1) or (3). If we are in case (1), $X^{\circ}$ acts as $B_{3}$ on $W^{\perp}$, so $u$ acts by an inner automorphism on a component of $X^{\circ}$ and we are done by Lemma 2.9 and 22.

In case (3) of Proposition 4.2, we have $W^{\perp} / W=V_{1} \oplus V_{2}$, with preimages $\tilde{V}_{1}, \tilde{V}_{2}$ in $W^{\perp}$. Now $X^{\circ}$ acts non-trivially on both $\tilde{V}_{i}$, normalised by $u$, so $u$ has two Jordan blocks on each $\tilde{V}_{i}$, by Theorem 3.9. Counting fixed points on $\tilde{V}_{1}+\tilde{V}_{2}$ as in Case 1a, we reach a contradiction.

Case 2. Now assume that $X^{\circ}$ acts trivially on $W$. Let $W_{0}$ be the $u$-invariant subspace of $W$ of codimension 1 . Note that $W_{0}^{\perp} / W_{0}$ is non-degenerate and $u$ acts as a regular unipotent element of $\mathrm{SO}\left(W_{0}^{\perp} / W_{0}\right)$ by Lemma 2.8 and the image of $X$ lies in a proper parabolic subgroup of this orthogonal group. So it suffices to derive a contradiction in that situation, whence henceforth we assume $\operatorname{dim} W=1$. Again, as $\operatorname{dim}\left(W^{\perp} / W\right) \geq 6$, we may apply the conclusion of Proposition 4.2 to the image of $X$ in $\mathrm{SO}\left(W^{\perp} / W\right)=\mathrm{SO}_{2 l-2}$ (using our assumption that $\operatorname{dim} W=1$ ).

Case 2a. In case (1) of Proposition 4.2 again we have $X=X^{\circ}$ as in Case 1b, a situation that was handled in [22].

Case 2b. In case (2) of Proposition 4.2 we have $X^{\circ}=A_{l-2}$, with $l \geq 4$ as $\operatorname{dim}\left(W^{\perp} / W\right) \geq 6$. Note that $V$ is then a completely reducible $X^{\circ}$-module since there are no extensions between the natural and the trivial module for $A_{l-2}$, so $W^{\perp} / W$ is isomorphic to an $X^{\circ}$-submodule $M$ of $W^{\perp}$. Assume that $M \cap M^{\perp} \neq 0$. Then, by dimension reasons, this intersection must be one of the two non-isomorphic irreducible $X^{\circ}$-summands. But then $M /\left(M \cap M^{\perp}\right)$ has a non-degenerate $X^{\circ}$ invariant form and thus is a self-dual $A_{l-2}$-module, which it is not. Thus, $M$ is a non-degenerate $X^{\circ}$-submodule of $V$, and $M^{\perp}$ is its 2-dimensional orthogonal complement. Since $M, M^{\perp}$ are both sums of homogeneous $X^{\circ}$-components of $V$, the decomposition $V=M \perp M^{\perp}$ is $u$-invariant, making $M$ an irreducible $X$-module. Thus $W \leq M^{\perp}$ is a 1-dimensional totally singular subspace, but the fixed points of the non-trivial unipotent elements of $\mathrm{GO}\left(M^{\perp}\right)=\mathrm{GO}_{2}$ are non-singular.

Case 2c. In case (3) of Proposition 4.2, we have an $X$-stable decomposition $W^{\perp} / W$ $=V_{1} \oplus V_{2}$ with $X$ irreducible on $V_{1}$. Write $\tilde{V}_{i}$ for the full preimage of $V_{i}$ in $W^{\perp}$, $i=1,2$, both $X$-submodules of $V$. By Theorem 3.9, as $\tilde{V}_{1}$ is reducible for $X$, we deduce that $u$ has two blocks on $\tilde{V}_{1}$. If $u$ has more than one Jordan block on $\tilde{V}_{2}$ as well, then counting fixed points as in Case 1a we obtain a contradiction. So $u$ has a single Jordan block on $\tilde{V}_{2}$, whence $\tilde{V}_{2}^{u}=W$ and $\left(W^{\perp}\right)^{u}=\tilde{V}_{1}^{u}$.

Now first assume that $p \neq 2$ so that $\operatorname{dim} V_{2}=1$. Using that $W^{\perp}=\tilde{V}_{1}+\tilde{V}_{2}$ we have $W^{\perp} /\left(W^{\perp}\right)^{u}=W^{\perp} / \tilde{V}_{1}^{u} \cong \tilde{V}_{1} / \tilde{V}_{1}^{u}+\tilde{V}_{2} / W$, and a dimension count then shows the sum is direct. Hence $\operatorname{dim}\left(W^{\perp} /\left(W^{\perp}\right)^{u}\right)^{u} \geq 2$, contradicting the Jordan block structure of $u$ on $V$. Similarly, when $p=2$ and hence $\operatorname{dim} V_{2}=2$, consider $M:=\operatorname{ker}\left(\left.(u-1)^{2}\right|_{W^{\perp}}\right)$. Then $\operatorname{dim} M \cap \tilde{V}_{1} \geq 3, \operatorname{dim} M \cap \tilde{V}_{2}=2$, and these two intersect in $W$. By assumption, $u$ has one fixed point on $V / \operatorname{ker}\left((u-1)^{2}\right)$, but on $W^{\perp} / M=\left(\tilde{V}_{1}+M\right) / M \oplus\left(\tilde{V}_{2}+M\right) / M$ it has a two-dimensional fixed point space, giving a contradiction. 
Case 2d. In case (4) of Proposition 4.2, we have $p=2$ and $W^{\perp} / W$ has $X$-invariant subspaces $0<\bar{V}_{1}<\bar{V}_{1}^{\perp}$ with $\bar{V}_{1}$ non-singular of dimension 1 , such that $u$ acts with one Jordan block on $\bar{V}_{1}^{\perp} / \bar{V}_{1}$. By Proposition 4.3 we may decompose $X^{\circ}=$ $X_{1} \cdots X_{r}$ into a product of simple groups all isomorphic to either $B_{m}, C_{m}$ or $G_{2}$, with $\operatorname{dim} V=2 l=2 m r+4$, where we set $m:=3$ in the case of $G_{2}$. Furthermore, $r$ is a power of 2 , with $r \geq 2$ since otherwise we are done by Lemma 2.9 and Proposition 4.5. As $u$ has Jordan blocks of sizes $2 m r+2,2$ on $V$, $u^{2}$ has two Jordan blocks of size $m r+1$ and two of size 1 .

Note that $v:=u^{2}$ acts trivially on the 2-dimensional full preimage $V_{1}$ of $\bar{V}_{1}$ in $V$. By our assumption, $V_{1}$ cannot be totally singular. Let $N \leq V_{1}$ be a 1 dimensional non-singular subspace. Then $X^{\circ}\langle v\rangle$ lies in the stabiliser $N_{\mathrm{SO}(V)}(N)$ isomorphic to $B_{l-1} \cong C_{l-1}$, so in $\operatorname{Sp}\left(N^{\perp} / N\right)$. We claim that $v$ has Jordan block sizes $m r+1, m r+1$ on $N^{\perp} / N$. Indeed, as it has block sizes $m r+1, m r+1,1,1$ on $V$, the only other possibility would be $m r, m r, 1,1$ (note that all odd block sizes must occur an even number of times). But by [9, Thm 6.6] the centraliser of such an element has reductive part of its centraliser containing an $\mathrm{Sp}_{2}$, while the reductive part of the centraliser of $v$ in $\mathrm{SO}(V)$ is just a torus, a contradiction.

Now the $X^{\circ}\left\langle v^{r / 2}\right\rangle$-composition factors of $N^{\perp} / N$ are the $U_{i}$ and two trivial modules. Thus, by self-duality, $N^{\perp} / N$ has a submodule $M$ of codimension 1 , and this is the sum of submodules of dimension at most $2 m+1$ (namely either the $U_{i}$ or extensions of some $U_{i}$ by a trivial module). But $v^{r / 2}=u^{r}$ has block sizes $2 m+1,2 m+1,2 m(r-2$ times $)$ on $N / N^{\perp}$, so at least one block of size $2 m+1$ on $M$. By Theorem 3.9, this contradicts the fact that $M$ has no irreducible $X^{\circ}\left\langle v^{r / 2}\right\rangle$ submodules of that dimension.

\section{EXCEPTIONAL TYPES}

In this section we consider algebraic groups defined over $k$ of characteristic $p>0$. See Remark 2.1

We will make extensive use of the known data on unipotent elements in simple algebraic groups of exceptional type, including element orders and power maps given in [6] and structure of centralisers described in [9. We follow the notation in [6] for the labelling of unipotent classes. In particular, if the class of $u$ is denoted by some Dynkin type, then $u$ is a regular element in a Levi subgroup of that type.

In the course of our proof we will require precise knowledge on the existence and conjugacy classes of complements to $R_{u}\left(C_{G}(x)\right)$ in $C_{G}(x)^{\circ}$, for certain unipotent elements $x$, as in the next result.

Lemma 5.1. Let $x \in G$ be unipotent and let $Y \leq C_{G}(x)$ be connected reductive, where

$$
(G, p, Y,[x]) \in\left\{\left(E_{7}, 3, A_{1}^{3}, 4 A_{1}\right),\left(E_{8}, 2, A_{2}, E_{6}\left(a_{1}\right)\right),\left(E_{8}, 2, A_{2}, E_{6}\right)\right\} .
$$

Then there exists a connected reductive group $C \leq C_{G}(x)$ such that $C_{G}(x)^{\circ}=$ $R_{u}\left(C_{G}(x)\right)$.C and $Y$ lies in a conjugate of $C$.

Proof. Throughout we write $R:=R_{u}\left(C_{G}(x)\right)$. The existence of a complement to $R$ in $C_{G}(x)^{\circ}$ follows from $[9,17.6]$. We now turn to the proof of the remaining assertions. 
Consider first $G=E_{7}$, with $p=3, Y=A_{1}^{3}, x$ a unipotent element of type $4 A_{1}$ and $Y \leq C_{G}(x)^{\circ}=R C$, where $C$ is a long root $C_{3}$-subgroup of $G$ (see [9, Tab. 22.1.2]). By [7, Thm 5] there exist two classes of such $C_{3}$-subgroups in $G$, coming from the two non-conjugate $A_{5}$ Levi factors of $G$. By [7, Cor., p.2], there exists a unique class of complements to $R$ in $R C$. For both classes of $C_{3}$-subgroups, each non-trivial $C$-composition factor of $R$ occurs as a composition factor of $\wedge^{j}(W)$, for some $1 \leq j \leq 3$, where $W$ is the natural 6 -dimensional $C$-module. (See 7 , Tab. 8.2].) There exists a unique class of $A_{1}^{3}$-subgroups of $C_{3}$, and restricting each of the given irreducible $C_{3}$-modules to such an $A_{1}^{3}$-subgroup we find that the composition factors of $Y$ on $R$ have highest weights among $\omega_{i}, i=1,2,3$ (the fundamental dominant weights of $\left.A_{1}^{3}\right), \omega_{i}+\omega_{j}$ for $1 \leq i<j \leq 3, \omega_{1}+\omega_{2}+\omega_{3}$ and the zero weight. Using [4, II.2.14] we have $H^{1}(Y, \bar{Q})=0$ for all $Y$-composition factors $\bar{Q}$ of $R$ and hence by [20, Prop. 3.2.6], there exists a unique class of complements to $R$ in $R Y$. Thus there exists $g \in C_{G}(x)$ with $Y \leq C^{g}$ as claimed.

In the other two cases, we have $G=E_{8}$, with $p=2, Y=A_{2}$, and $x$ is a unipotent element of type either $E_{6}\left(a_{1}\right)$ or $E_{6}$, and $C_{G}(x)^{\circ}=R C$, where $C=\bar{A}_{2}$, respectively $\bar{G}_{2}$, long root subgroups. The action of a long root $A_{2}$ on $R$ has composition factors the natural, dual or trivial module, and so in case $C=\bar{A}_{2}$, we have a unique class of complements to $R$ in $R Y=R C$, establishing the result.

In the case $C=\bar{G}_{2}$ we must argue slightly differently because here there is a 6-dimensional $C$-composition factor $V$ of $R$ with $H^{1}\left(G_{2}, V\right) \neq 0$. Now $C_{G}(x)$ lies in a parabolic subgroup $P=Q L$ of $G$ with $R \leq Q$. Moreover, considering the labelled diagram of the class of $x$ (see [9, Tab. 22.1.1]) and applying [9, Thm 17.4], we see that we may take $P$ to be a $D_{4}$-parabolic subgroup of $G$. There exists a composition series of $Q$ as an $[L, L]$-module, all of whose terms are 8-dimensional $D_{4}$-modules and trivials. The subgroup $C$ is uniquely determined up to conjugacy in $[L, L]$ and, by [8, Lemma 9.1.1], each such irreducible upon restriction to $C$ is the indecomposable tilting module $U$ with $\operatorname{rad} U / \operatorname{Soc} U$ the 6 -dimensional irreducible $C$-module. By [4, Prop. $\S$ E.1], $H^{1}(C, U)=0$. Hence, all complements to $C$ in $Q C$ are conjugate and by considering the action of $Y=A_{2}$ on $Q$, we have the same statement for $Y$. Arguing as in the previous cases now yields the claim.

Theorem 5.2. Let $G$ be a simple algebraic group of exceptional type and $X=$ $X^{\circ}\langle u\rangle \leq G$ a reductive subgroup with $u$ a regular unipotent element of $G$ and $\left[X^{\circ}, X^{\circ}\right] \neq 1$. Then $X$ does not lie in a proper parabolic subgroup of $G$.

Proof. Let $X$ be as in the assertion and assume that $X$ lies in a proper parabolic subgroup of $G$. Then we have $u \notin X^{\circ}$ by [22, Thm 1.2]. Also, by passing to $\left[X^{\circ}, X^{\circ}\right]\langle u\rangle$ we may assume that $X^{\circ}$ is semisimple. We will need to consider the image of $X$ in Levi factors, and for this throughout we write $\bar{X}$ for the quotient of $X$ by its largest normal unipotent subgroup (which, being finite, is centralised by $X^{\circ}$ ). Note that $X^{\circ}$ maps isomorphically to a subgroup of $\bar{X}$ on which the image of $\langle u\rangle$ then acts faithfully.

By Remark 2.2, we may moreover assume that $u$ has a single orbit on the set of simple components of $X^{\circ}$, and, by Lemma 2.9 it does not act as an inner automorphism on $X^{\circ}$. In particular, $\operatorname{rnk}\left(X^{\circ}\right) \geq p$. On the other hand, as $X$ lies in a proper parabolic subgroup of $G, \operatorname{rnk}\left(X^{\circ}\right)<\operatorname{rnk}(G)$. This already rules out the case $G=G_{2}$. Furthermore, regular unipotent elements of $F_{4}$ are also regular in $E_{6}$ under the natural embedding, and proper parabolic subgroups of $F_{4}$ lie in such of 
$E_{6}$, hence it suffices to prove our result for $G=E_{6}, E_{7}$ or $E_{8}$. The orders of regular unipotent elements in these groups for small primes are given in Table 2.

TABLE 2. Orders of regular unipotent elements

\begin{tabular}{c|cccc} 
& $p=2$ & 3 & 5 & 7 \\
\hline$G_{2}$ & 8 & 9 & & \\
$F_{4}$ & 16 & 27 & & \\
$E_{6}$ & 16 & 27 & 25 & \\
$E_{7}$ & 32 & 27 & 25 & 49 \\
$E_{8}$ & 32 & 81 & 125 & 49
\end{tabular}

Case 1. We first consider the case that $v:=u^{p}$ acts by an inner automorphism on $X^{\circ}$ and $X^{\circ}$ does not contain elements of order $|v|$, so in particular $|v|>p$.

Then by Lemma 3.5, $X^{\circ}$ is centralised by a unipotent element $v^{\prime}$ of this order. We discuss this situation by comparing the list of centralisers of unipotent elements [9, §22] and the list of unipotent element orders [6, Tab. 5-9].

Let first $G=E_{6}$ and consider the case $p=2$, where we have $\left|v^{\prime}\right|=|v|=8$ and $v^{\prime}$ centralises $X^{\circ}$. By [9, Tab. 22.1.3] and [6, Tab. 5] unipotent elements of order 8 centralising a group of semisimple rank at least 2 lie in class $D_{4}$, with reductive part of the centraliser of type $A_{2}$. Since $\operatorname{rnk}\left(X^{\circ}\right) \geq 2$ we conclude that $X^{\circ}=A_{2}$. Now $u^{2}$ acts as an inner automorphism of $X$ and so $X$ centralises $u^{8}$, which lies in the class $2 A_{1}$, by [6, Tab. D]. Moreover, using [9, Tab. 22.1.3], we see that the full connected centraliser of $u^{8}$ has a reductive complement $C$ to $R=R_{u}\left(C_{G}\left(u^{8}\right)\right)$, a $B_{3}$-subgroup of $G$ generated by long root elements of $G$. Now $X^{\circ} \leq R C$ and we consider the possible embedding of $X^{\circ}$ in $C \cong C_{G}\left(u^{8}\right)^{\circ} / R$. By [10, Lemma 2.2], $X$ must lie in a proper parabolic subgroup of $C$ and by rank considerations we find that $X$ lies in an $A_{2}$-parabolic subgroup of $C$. As $C$ is generated by long root subgroups of $G$, the Levi factor $A_{2}$ is also generated by long root subgroups of $G$. Now arguing as in Lemma 5.1, we find that $X^{\circ}$ is a long root $A_{2}$-subgroup of $G$, that is, a Levi factor of $G$. (The $A_{2}$ Levi factor of $C$ acts on $R$ with composition factors the natural, dual or trivial module for $A_{2}$.) But now the centraliser of $X^{\circ}$ is an $A_{2} A_{2}$ and so $X$ normalizes an $A_{2}^{3}$ subgroup of $G$. But there is no such example in [17, Thm A]. For $p=3$ we have $\left|v^{\prime}\right|=9$ and $\operatorname{rnk}\left(X^{\circ}\right) \geq 3$, and again by [9, Tab. 22.1.3] and [6, Tab. 5] there is no possibility. For $p=5$ we have $\left|v^{\prime}\right|=5$, contrary to our assumption.

For $G=E_{7}$ and $p=2$ we have $\left|v^{\prime}\right|=16$ but all centralisers of such elements have semisimple rank at most 1 by [9, $\S 22]$. When $p=3$ and so $\left|v^{\prime}\right|=9$, the unipotent classes $A_{3},\left(A_{3}+A_{1}\right)^{(1)},\left(A_{3}+A_{1}\right)^{(2)}, D_{4}$ and $D_{4}\left(a_{1}\right)$ need to be discussed. Here the semisimple parts of the centralisers have type $B_{3} A_{1}, B_{3}, A_{1}^{3}, C_{3}, A_{1}^{3}$, respectively. As $X^{\circ}$ is contained in one of those, and $u$ has a single orbit on its set of simple components, $X^{\circ}$ must be of type $A_{1}^{3}$. Now $X=A_{1}^{3}\langle u\rangle$ contains $u^{3}$ acting as an inner element on $A_{1}^{3}$, and so $u^{9}$ centralises $A_{1}^{3}$. But by [6, Tab. D], $u^{9}$ lies in class $4 A_{1}$, with semisimple part of its centraliser a $C_{3}$-subgroup generated by long root subgroups, by [9, §22]. By Lemma 5.1 $X^{\circ}$ must be a long root $A_{1}^{3}$. There are two classes of Levi subgroups $A_{1}^{3}$ in $E_{7}$. Using Borel-de Siebenthal one sees that one is centralised by an $A_{1}^{4}$, the other by a $D_{4}$. Thus in any case, $X^{\circ} C_{G}\left(X^{\circ}\right)$ contains 
a subgroup of $E_{7}$ of maximal semisimple rank, so the normaliser of $X^{\circ}$ and all of its overgroups are reductive. But by [17, Thm A] there are no such subgroups containing a regular unipotent element. For $p=5$ we have $\left|v^{\prime}\right|=5$, contrary to our assumption.

Finally, assume $G=E_{8}$. For $p=2$ we have $\left|v^{\prime}\right|=16$. Only the 17 unipotent classes

$$
\begin{gathered}
E_{6}\left(a_{1}\right), D_{6}, E_{6}, E_{6}\left(a_{1}\right)+A_{1}, E_{7}\left(a_{3}\right), E_{8}\left(b_{6}\right), D_{7}\left(a_{1}\right), D_{7}\left(a_{1}\right)^{(2)}, E_{6}+A_{1}, \\
E_{7}\left(a_{2}\right), E_{8}\left(a_{6}\right), D_{7}, E_{8}\left(b_{5}\right), E_{7}\left(a_{1}\right), E_{8}\left(a_{5}\right), E_{8}\left(b_{4}\right), E_{8}\left(a_{4}\right)
\end{gathered}
$$

of $G$ contain elements of order 16 . Of these, only the first three cases have a semisimple part of the centraliser of rank at least 2, of type $A_{2}, B_{2}$ and $G_{2}$ respectively. So $X^{\circ}=A_{2}$ or $A_{1}^{2}$, with $u$ acting by the graph automorphism. In the second case, $u^{4}$ centralises $X^{\circ}$ and lies in class $D_{7}\left(a_{2}\right)$, but the latter has centraliser of rank 1 . So in fact $X^{\circ}=A_{2}$. By [9, Tab. 22.1.1], the remaining possible subgroups $A_{2}, G_{2}$ of $C_{G}\left(v^{\prime}\right)$ are generated by long root subgroups, and by Lemma $5.1, X^{\circ}$ is contained in one of them. Now all subgroups of type $A_{2}$ of these are again generated by long root subgroups, hence so is $X^{\circ}$. Thus, the centraliser of $X^{\circ}$ is of type $E_{6}$, and so $u$ acts on an $E_{6} A_{2}$, whose normaliser is a maximal subgroup of $E_{8}$. But since this does not appear in [17, Thm A], its normaliser does not contain regular unipotent elements.

If $p=3$ then $\left|v^{\prime}\right|=27$, but no unipotent element of order bigger than 9 has a centraliser of semisimple rank at least 3. If $p=5$ then $\left|v^{\prime}\right|=25$, but none of the seven unipotent classes having centraliser of semisimple rank at least 5 contains elements of order 25. Finally, for $p=7$ we have $\left|v^{\prime}\right|=7$, which is not allowed here.

Case 2. We next consider the case that $v:=u^{p}$ acts by an inner automorphism on $X^{\circ}$, and that $X^{\circ}$ does contain an element of order $|v|$.

When $G=E_{6}$ and $p=2$, then $X^{\circ}$ is a semisimple subgroup with an element of order 8 having a non-trivial graph automorphism transitively permuting the simple factors. Therefore, $X^{\circ}$ is one of $A_{4}, A_{5}, D_{4}, D_{5}, G_{2}^{2}$. By assumption $X$ lies in a proper parabolic subgroup of $G$ with Levi factor $L$, and thus $L$ contains one of the above groups, with the image of $u$ inducing a non-trivial graph automorphism of order 2. By rank considerations, only $X^{\circ}=A_{4}, D_{4}$ and $G_{2}^{2}$ might occur. The minimal dimension of a representation of $\bar{X}=A_{4} .2$ on which $A_{4}$ acts non-trivially is 10 , so this cannot occur inside $A_{5}$. This representation embeds $A_{4} .2$ into $\mathrm{GO}_{10}$, but not into $\mathrm{SO}_{10}$ by the block structures given in Lemma 2.12. Also, the smallest faithful representation of $G_{2}^{2}$ has dimension 12, too large for any proper Levi subgroup. So in fact we must have $X^{\circ}=D_{4}$ inside a $D_{5}$-parabolic. Using [20, 3.2.6] one can check that this embedding is into a Levi factor, and so $X^{\circ}=D_{4}$ is a Levi subgroup of $G$. By [9, Tab. 22.1.3], no non-trivial unipotent element of $G$ has a $D_{4}$ in its centraliser, so $C_{G}\left(X^{\circ}\right)$ is a torus, and then in fact it must be the centre $T_{2}$ of a Levi subgroup of type $D_{4}$. As $u$ acts on $C_{G}\left(X^{\circ}\right)$, Proposition 7.8 below implies that $u^{4}$ must centralise $T_{2}$. But $u^{4}$ lies in the class $2 A_{2}+A_{1}$ and has centraliser of rank 1 by [9, Tab. 22.1.3], a contradiction.

For $G=E_{6}$ and $p=3$ with $|v|=9$, we have that $X^{\circ}$ contains elements of order 9 and has an outer automorphism of order 3 , so $\bar{X}=D_{4} .3$. But the smallest faithful representation of $X$ has dimension 24, which is too large for containment in any proper parabolic subgroup of $G$. For $p=5$ where $|v|=5$ the only option is that 
$X^{\circ}=A_{1}^{5}$. But again by Borel-de Siebenthal no proper parabolic subgroup has a Levi factor containing a group $X$ with $\bar{X}=A_{1}^{5} .5$.

For $G=E_{7}$ with $p=2$ the semisimple group $X^{\circ}$ has an element of order 16 and a non-trivial graph automorphism, whence $X^{\circ} \in\left\{D_{6}, E_{6}\right\}$. But clearly no Levi factor of a proper parabolic subgroup of $E_{7}$ can contain $X$ with $\bar{X}=D_{6} .2$ or $E_{6} .2$. For $p=3$ with $|v|=9$ the only possibilities with a graph automorphism of order 3 are $\bar{X}=D_{4} .3, B_{2}^{3} .3$ and $G_{2}^{3} .3$. All could only lie in a proper parabolic subgroup of type $E_{6}$. But $E_{6}$ has no maximal rank subgroups $B_{2}^{3}$ or $G_{2}^{3}$ by Borel-de Siebenthal. When $\bar{X}=D_{4} .3,\left[7\right.$, Thm 5] shows that $X^{\circ}$ is a Levi factor of $G$. Again by Borel-de Siebenthal there is a subgroup $A_{1}^{3}$ centralising $X^{\circ}=D_{4}$, so $N_{G}\left(D_{4}\right) \geq D_{4} A_{1}^{3}$ is reductive. But by [17, Thm A], there is no positive-dimensional maximal reductive subgroup of $G$ containing a regular unipotent element. For $p=5$ again the only possibility is $X^{\circ}=A_{1}^{5}$. The only proper parabolic subgroups whose Levi factor might contain $X$ with $\bar{X}=A_{1}^{5} .5$ are those of type $D_{6}$. The list in [17, Thm B] shows that there is no maximal reductive subgroup of $D_{6}$ containing a regular unipotent element of $D_{6}$ and such an $A_{1}^{5}$.

For $G=E_{8}$ and $p=2$ we have $|v|=16$. The only semisimple groups of rank at most 7 with a unipotent element of order 16 and an even order graph automorphism are $D_{6}, D_{7}$ and $E_{6}$. Now for $X^{\circ}=D_{6}$ or $D_{7}$ the element $v$ of order 16 acts as an inner element $x$ of order 8 . Thus $X^{\circ}$ is centralised by the element $v x^{-1}$ of order 16 , which is not possible. Assume $X^{\circ}=E_{6}$ and $u$ induces a graph automorphism on $E_{6}$. There is only one class of subgroups $E_{6}$ in $E_{8}$ by [7, Thm 5] and hence $X^{\circ}$ is a Levi factor of $G$. Again, $u$ normalises the centraliser of such an $E_{6}$, hence a subgroup $E_{6} A_{2}$, and as above this is not possible by [17, Thm A]. If $p=3$ then $|v|=27$, and there is no possible case. When $p=5$ or $p=7$, then $X^{\circ}$ must have at least $p$ simple components, whence $X^{\circ}=A_{1}^{p}$. Now for $p=5$, the group $A_{1}^{5}$ does not contain elements of order 25 , so we have $p=7$ and $X^{\circ}=A_{1}^{7}$. The only proper parabolic subgroup of $E_{8}$ with a Levi factor containing $X$ with $\bar{X}=A_{1}^{7} .7$ is of type $E_{7}$. By [11, Thm 4 and Tab. 17 and 18], such an $A_{1}^{7}$ lies in a Levi factor $E_{7}$. Now the centraliser of that $A_{1}^{7}$ contains the $A_{1}$ centralising the $E_{7}$-Levi subgroup. So again the normaliser of $X^{\circ}$ in $E_{8}$ has maximal semisimple rank, and [17, Thm A] shows that this cannot contain regular unipotent elements.

Case 3. Finally, consider the case that $u^{p}$ is not inner. Then either $X^{\circ}$ has at least $p^{2}$ components, or there are $p$ components and on each of them $u^{p}$ induces a graph automorphism of order $p$. Either possibility forces $\operatorname{rnk}\left(X^{\circ}\right) \geq p^{2}$, so $p=2$. Furthermore, $v:=u^{4}$ must act by an inner automorphism on $X^{\circ}$. When $G=E_{6}$ then the possibilities are $X^{\circ}=A_{1}^{4}$ or $A_{2}^{2}$. In the first case, by Lemma 3.5 there exists an element of order 4 centralising an $A_{1}^{4}$, which is not possible by [9, §22]. In the case $X^{\circ}=A_{2}^{2}$ and $u$ acts as an outer automorphism of order 4 . The only Levi factor possibly containing a subgroup $X$ with $\bar{X}=A_{2}^{2} .4$ is of type $D_{5}$, but $A_{2}^{2} .4$ contains elements of order 16 while $D_{5}$ does not have such elements. When $G=E_{7}$ then $|v|=8$; none of the groups $A_{1}^{4}, A_{2}^{2}$ and $A_{3}^{2}$ contains elements of that order, so by Lemma 3.5 there is an element of $G$ of order 8 centralising such a subgroup, which is not the case by [6] and [9]. Finally, when $G=E_{8}$ then again $|v|=8$, and the candidates for $X^{\circ}$ are $A_{1}^{4}, A_{2}^{2}$ and $A_{3}^{2}$. Assume $\bar{X}=A_{1}^{4} \cdot 4$, then $u^{4}$ acts by an inner automorphism so $u^{8}$ centralises $X^{\circ}$. But $u^{8}$ lies in class $D_{4}\left(a_{1}\right)+A_{2}$ (there is a misprint in [6. Tab. D]), and its centraliser does not contain an $A_{1}^{4}$. Similarly, if $\bar{X}=A_{2}^{2} .4$ then $u^{16}$, in class $4 A_{1}$, centralises $X^{\circ}$, which is not possible. The same 
argument rules out $\bar{X}=A_{3}^{2} .4$. This completes our case distinction and thus the proof.

Theorem 1 now follows by combining Theorems 3.9 , 4.7 and 5.2 .

\section{Regular Unipotent ElEMENTS in ALmost Simple groups}

We now extend our main result to the case of regular unipotent elements in cosets of simple groups in almost simple groups.

Example 6.1. The regular unipotent elements in a coset $x G^{\circ} \neq G^{\circ}$ of an almost simple group $G$ of "exceptional type" can be realized as follows:

(a) The group $D_{4} .3$ occurs as a subgroup of $F_{4}$ in a natural way (see e.g. [15, Ex. 13.9]). Now according to [6, Tab. 4] the only unipotent class of $F_{4}$ for $p=3$ containing elements of order 27 is the class of regular unipotent elements. Also, the regular unipotent elements in an outer coset of the disconnected group $D_{4} .3$ have order 27 (see [13, Tab. 8]). Thus, they are regular unipotent elements of $F_{4}$.

(b) Similarly, the disconnected group $E_{6} .2$ occurs inside the normaliser of a Levi subgroup of type $E_{6}$ inside $E_{7}$. Again by [6, Tab. 7] the only unipotent class of $E_{7}$ for $p=2$ containing elements of order 32 is the class of regular unipotent elements, and since regular unipotent elements in the outer coset of $E_{6} .2$ have order 32 (see [14, Tab. 10]), they must be regular unipotent elements of $E_{7}$.

We then obtain the following consequence of Theorem 1

Corollary 6.2. Let $G$ be almost simple of type $A_{l} .2, D_{l} .2$ or $E_{6} .2$ with $p=2$, or of type $D_{4} .3$ with $p=3$, and $X=X^{\circ}\langle u\rangle \leq G$ be a reductive subgroup with $u$ a regular unipotent element of $u G^{\circ}$ and $\left[X^{\circ}, X^{\circ}\right] \neq 1$. Then $X$ does not lie in any proper subgroup $P$ of $G$ such that $P^{\circ}$ is a parabolic subgroup of $G^{\circ}$.

Proof. In each case, we embed $G$ in a simple algebraic group $H$; namely, $A_{2 l} .2$ and $D_{l} .2$ embed into $H:=\mathrm{SL}(V)$ via their natural representation, $G=A_{2 l-1} .2$ embeds into $H:=D_{2 l}$ (see remarks before Lemma 2.12), and $D_{4} .3, E_{6} .2$, embed into $H=F_{4}, E_{7}$ respectively under the embeddings given in Example 6.1. Applying Lemma 2.12 and Example6.1, we have that the embedding sends regular unipotent elements in an outer coset of $G^{\circ}$ to regular unipotent elements of $H$. Now, if $X$ lies in a proper subgroup $P$ of $G$ with $P^{\circ}$ a parabolic subgroup of $G^{\circ}$ with $Q=R_{u}\left(P^{\circ}\right)$, then $X \leq N_{H}(Q)$, and by the Borel-Tits theorem, the latter lies in a proper parabolic subgroup of $H$. Thus, in all cases our claim for the almost simple group $G$ follows from Theorem 1 for the simple group $H$.

Note that the type of subgroups $P$ allowed for in the preceding statement are those given by the most general possible definition of "parabolic subgroups of an almost simple group".

\section{Regular unipotent elEMENTS IN NORMALISERS OF TORI}

Here, we show that if one removes the hypothesis that $\left[X^{\circ}, X^{\circ}\right] \neq 1$ in Theorem 1 , the conclusion is no longer valid. More generally, for a simple group $G$ we investigate the structure of torus normalisers in $G$ that contain a regular unipotent element and lie in some proper parabolic subgroup of $G$. 


\subsection{Torus normalisers in $\mathrm{SL}(V)$.}

Proposition 7.1. Let $X=T\langle u\rangle \leq \mathrm{SL}(V)$ where $T$ is a torus and $u$ is unipotent with a single Jordan block. Then all weight spaces of $T$ on $V$ have the same dimension d. Moreover $X$ is contained in a proper parabolic subgroup of $\mathrm{SL}(V)$ if and only if $d>1$.

Proof. Since $u$ normalises $T$, the weight spaces of $T$ on $V$ are permuted by $u$. Moreover this action must be transitive as otherwise $u$ would have at least two Jordan blocks on $V$. Thus, they all have the same dimension, and if they are 1dimensional, $V$ is an irreducible $X$-module and so $X$ does not lie in any proper parabolic subgroup of $G$.

Now assume the common dimension of the weight spaces is $d>1$ and set $m=$ $\operatorname{dim}(V) / d$. Since $u$ has $p$-power order, the number of weight spaces, $m$, is a $p$-power. It follows by Lemma 2.3 that $u^{m}$ acts with a single Jordan block (of size $d$ ) on each weight space. In particular, $T$ centralises $u^{m} \neq 1$ and thus $X \leq C_{G}\left(u^{m}\right)$ lies in a proper parabolic subgroup of $G$ by the Borel-Tits theorem ([15, Rem. 17.16]).

Groups as in the previous result do in fact exist:

Proposition 7.2. Let $n=p^{a} d$ be an integer, where $a>0$. There exists $a\left(p^{a}-1\right)$ dimensional torus $T \leq \mathrm{SL}(V)$, where $\operatorname{dim} V=n$, with d-dimensional weight spaces on $V$, normalised by a unipotent element $u \in \mathrm{SL}(V)$ with a single Jordan block. Moreover, $T\langle u\rangle$ lies in a proper parabolic subgroup of $\mathrm{SL}(V)$ if and only if $d>1$.

Proof. Decompose $V=V_{1} \oplus \cdots \oplus V_{m}$ into a direct sum of $m:=p^{a}$ subspaces of dimension $d$. Let $x \in \mathrm{SL}(V)$ be the permutation matrix for a permutation sending an ordered basis of $V_{i}$ to an ordered basis of $V_{i+1}$ for $i=1, \ldots, m$, where $V_{m+1}:=V_{1}$. Then $x$ has order $m$. For $i=1, \ldots, m$ let $T_{i} \leq \operatorname{GL}\left(V_{i}\right)$ be the torus of scalar matrices and $u_{1} \in \mathrm{SL}\left(V_{1}\right)$ a unipotent element with a single Jordan block. Set $X^{\prime}:=\left\langle T_{1}, u_{1}, x\right\rangle \leq \mathrm{GL}(V)$. As $x$ permutes the $T_{i}$ transitively and $x^{m}=1$ we have that $X^{\prime}$ is the wreath product of $T_{1} \times\left\langle u_{1}\right\rangle$ with $\langle x\rangle$, and we can write elements of $X^{\prime}$ as $\left(x_{1}, \ldots, x_{m} ; x^{j}\right)$ for $x_{i} \in\left(T_{1}\left\langle u_{1}\right\rangle\right)^{x^{i-1}}$ and some $j$. Then the element $u:=\left(u_{1}, 1, \ldots, 1 ; x\right)$ has $m$ th power $u^{m}=\left(u_{1}, \ldots, u_{1} ; 1\right)$ which has $m$ Jordan blocks of size $d$ on $V$. But then $u$ must have a single Jordan block on $V$ by Lemma 2.3. Now with $T:=T_{1} \cdots T_{m} \cap \mathrm{SL}(V)$ the subgroup $T\langle u\rangle$ is as in Proposition 7.1 and thus the claim follows.

7.2. Torus normalisers in $\operatorname{Sp}(V)$ and $\mathrm{GO}(V)$. We next discuss those classical groups in which regular unipotent elements have a single Jordan block on the natural module, that is, the types $B_{l}, C_{l}$ and $D_{l} .2$ (see Lemma 2.12).

For this, note that weight spaces for non-zero weights of a torus $T$ in $\operatorname{Sp}(V)$ and $\mathrm{SO}(V)$ are totally isotropic and totally singular, respectively, and weight spaces for weights $\chi, \eta$ with $\chi \neq-\eta$, are orthogonal to each other. To see this for $\mathrm{SO}(V)$, let $Q$ be the quadratic form and $\beta$ the associated bilinear form on $V$. Let $\chi \neq 0$ be a weight of $T$ with weight space $V_{\chi}$. Then for $v \in V_{\chi}$, we have $Q(v)=Q(t v)=$ $Q(\chi(t) v)=\chi(t)^{2} Q(v)$, for all $t \in T$. Since there exists $t \in T$ with $\chi\left(t^{2}\right) \neq 1$ we find $Q(v)=0$. So non-zero weight spaces are indeed totally singular. Further, for $\chi$ and $\eta$ two weights of $T$ and $v \in V_{\chi}, w \in V_{\eta}$, we have $\beta(v, w)=\beta(t v, t w)=$ $\chi(t) \eta(t) \beta(v, w)$ for all $t \in T$, and if $\beta(v, w) \neq 0$ then $\chi=-\eta$. The argument for $\operatorname{Sp}(V)$ is completely analogous. 
Lemma 7.3. Let $G=B_{l}$ or $C_{l}$ with $l \geq 1$ and $X=T\langle u\rangle \leq G$ where $T \neq 1$ is a torus and $u$ is regular unipotent in $G$. Then $p=2$.

Proof. Assume $p \neq 2$. Write $V$ for the natural module of $G$ and let $V=\bigoplus_{i=1}^{r} V_{i}$ be its $T$-weight space decomposition. Note that we have $r \geq 2$ since $Z(G)^{\circ}=1$. Now $u$ permutes the $V_{i}$ and hence their corresponding weights. As $u$ acts as a single Jordan block on $V$ by Lemma 2.12, this action must be transitive, so $r$ is a power of $p$. Since $r \geq 2$ there is at least one non-zero weight $\chi$. As we are in $\operatorname{Sp}(V)$ or $\mathrm{SO}(V)$, then $-\chi$ is also a weight, so both $\chi$ and $-\chi$ lie in one $u$-orbit, contradicting that $p \neq 2$.

In the case $p=2$, by the exceptional isogeny between $B_{l}$ and $C_{l}$ we need not consider type $B_{l}$.

Proposition 7.4. Let $p=2, G=\operatorname{Sp}(V)$ or $\mathrm{GO}(V)$ and $X=T\langle u\rangle \leq G$ with $T \neq 1$ a torus and $u$ having a single Jordan block on $V$. If $V=V_{1} \oplus \cdots \oplus V_{r}$ is the $T$-weight space decomposition then the $V_{i}$ are totally isotropic or totally singular, respectively, and permuted transitively by $u$. Moreover, up to renumbering, there is an orthogonal decomposition $V=\left(V_{1} \oplus V_{r / 2+1}\right) \perp \ldots \perp\left(V_{r / 2} \oplus V_{r}\right)$.

Proof. As $u$ has a single Jordan block, it permutes the $V_{i}$ transitively and so $r$ is a 2-power. All $V_{i}$ are totally isotropic or totally singular, respectively, by the remarks before Lemma 7.3, and orthogonal to all other weight spaces that do not have opposite weight. Further, if $\chi$ is a weight of $T$ then so is $-\chi$, and thus for a suitable numbering, $V_{i}$ and $V_{i+r / 2}$ have opposite weights, for $i=1, \ldots, r / 2$. Thus we obtain the claimed orthogonal decomposition.

Example 7.5. The situation nailed down in Proposition 7.4 does give rise to examples within proper parabolic subgroups. To see this, let $p=2, G=\operatorname{GO}(V)$ with $\operatorname{dim} V=2 l$, where $l=2^{f} m$ with $m>1$ odd. By Lemma 2.12 for any odd $m$ the stabiliser $\mathrm{GL}_{m} .2$ in $\mathrm{GO}_{2 m}$ of a maximal totally singular subspace contains a unipotent element $v$ with a single Jordan block. This normalises $T_{1}:=Z\left(\mathrm{GL}_{m}\right)$, and $v^{2} \neq 1$ centralises $T_{1}$. Now embed

$$
T_{1}\langle v\rangle \times \cdots \times T_{1}\langle v\rangle \leq H:=\mathrm{GO}_{2 m} \times \ldots \times \mathrm{GO}_{2 m} \leq \mathrm{GO}_{2 l}=\mathrm{GO}(V)
$$

( $2^{f}$ factors). The normaliser of $H$ in $\mathrm{GO}_{2 l}$ contains an element $x$ cyclically permuting the factors. Set $u:=(v, 1, \ldots, 1) x$. By construction, $u^{2^{f}}=(v, \ldots, v)$ has $2^{f}$ Jordan blocks of size $2 m$, so by Lemma 2.3. $u$ has a single Jordan block on $V$, it normalises $T:=T_{1}^{2^{f}}$, and $u^{2^{f+1}} \neq 1$ centralises $T$. Thus, $T\langle u\rangle$ lies in a proper parabolic subgroup of $\operatorname{GO}(V)$. Since $D_{l} .2 \leq C_{l}$, this also provides examples in $C_{l}$.

7.3. Torus normalisers in $\mathrm{SO}(V)$. Here we consider tori in $D_{l}$ normalised by a regular unipotent element.

Proposition 7.6. Let $X=T\langle u\rangle \leq \mathrm{SO}(V)$ with $\operatorname{dim} V=2 l \geq 8, T \neq 1$ a torus and $u$ regular unipotent in $\mathrm{SO}(V)$. Then $p=2$ and if $V=V_{1} \oplus \cdots \oplus V_{r}$ is the $T$-weight space decomposition then up to renumbering the $V_{i}$, we have one of:

(1) $r=2, l$ is even, $u$ interchanges $V_{1}$ and $V_{2}$, and $u^{2}$ acts with Jordan blocks of sizes $l-1,1$ on both $V_{1}$ and $V_{2}$;

(2) $u$ permutes $V_{1}, \ldots, V_{r-1}$ transitively (so $r=2^{s}+1$ for some $s \geq 1$ ) and $V_{r}$ is the 0-weight space, with $\operatorname{dim} V_{r}=2$; or 
(3) $\langle u\rangle$ acts transitively on $\left\{V_{1}, \ldots, V_{r-2}\right\}$ and on $\left\{V_{r-1}, V_{r}\right\}$, so $r=2^{s}+2$ for some $s \geq 0$, and $V_{r-1}$ and $V_{r}$ are 1-dimensional weight spaces for opposed weights.

Proof. Let $V=\bigoplus_{i=1}^{r} V_{i}$ be the decomposition of $V$ into non-zero $T$-weight spaces. Note that we have $r \geq 2$ since $Z(\mathrm{SO}(V))^{\circ}=1$. From the block structure of $u$ it follows that $\langle u\rangle$ has at most two orbits on the set of $V_{i}$. In addition, the sum of the weight spaces in one of the orbits is of dimension at most 2 . Since we are in $\mathrm{SO}(V)$, if $\chi$ is a weight of $T$ on $V$, then so is $-\chi$. Now first assume that $p$ is odd. Then $\chi$ and $-\chi$ can only lie in the same $u$-orbit if $\chi=0$. So $u$ has two orbits on the set of weight spaces, one of length $r-1$ and the other of length 1 . There is a non-zero weight $\chi$ in one of the orbits; the weight space of $-\chi$ then lies in the other orbit. This forces $\operatorname{dim} V=2$, contrary to our assumption.

Thus we have $p=2$. First assume $u$ permutes the $V_{i}$ transitively. Then $u^{r}$ stabilises each $V_{i}$, and has same block sizes $n_{1}, \ldots, n_{s}$ on each of them. Since $r$ is a 2-power, the blocks of $u$ on $V$ then have sizes $r n_{1}, \ldots, r n_{s}$, whence $r \leq 2$ and so $r=2$. Since $V_{1}$ and $V_{2}$ are both totally singular, $X$ is contained in the stabiliser of a decomposition of $V$ into a sum of two maximal totally singular subspaces. If $l$ is odd, then this stabiliser in $\mathrm{SO}(V)$ fixes each $V_{i}$ (see [5, Lemma 2.5.8]). Thus $l$ is even, $u$ interchanges $V_{1}$ and $V_{2}$ and $u^{2}$ has Jordan blocks as claimed in (1).

Next assume that $u$ permutes $V_{1}, \ldots, V_{r-1}$ transitively. Then without loss of generality $\operatorname{dim} V_{r}=2$. If $V_{r}$ is not the 0 -weight space, then the opposite weight space must be one of the other $V_{i}$, so $r=2$, and $\operatorname{dim} V=4$, contradicting our assumption. So we arrive at (2).

Finally, assume that $u$ permutes $V_{1}, \ldots, V_{r-2}$ transitively. Then $\operatorname{dim} V_{r-1}=$ $\operatorname{dim} V_{r}=1$ and the corresponding weights are opposed and interchanged by $u$, which is $(3)$

Example 7.7. We show that the cases in Proposition 7.6 do give rise to examples within proper parabolic subgroups. So let $p=2$.

(1) Let $l$ be even, $T$ be the 1-dimensional central torus of $\mathrm{GL}_{l}$ inside the stabiliser $\mathrm{GL}_{l} .2$ in $\mathrm{SO}_{2 l}$ of a pair $V_{1}, V_{2}$ of maximal totally singular subspaces. Thus $T$ acts by scalars on both $V_{1}, V_{2}$. Then $T$ is normalised by the outer elements of $\mathrm{GL}_{l} .2$ interchanging $V_{1}, V_{2}$. Now by Lemma 2.12, a regular unipotent element $u$ in the outer coset of $\mathrm{GL}_{l} .2$ has Jordan blocks of sizes $2 l-2,2$, hence is regular unipotent in $\mathrm{SO}_{2 l}$. Then $X:=T\langle u\rangle$ lies in the centraliser of the non-trivial unipotent element $u^{2} \in \mathrm{GL}_{l}$ (non-trivial as soon as $l-1 \geq 2$ ), thus inside a proper parabolic subgroup. This is an example of (1) in Proposition 7.6 .

(2) Let $H=\mathrm{GO}_{2 l-2} \mathrm{GO}_{2} \cap \mathrm{SO}(V)$ be the stabiliser of an orthogonal decomposition of $V$, where $\operatorname{dim} V=2 l$ with $l=2^{s}+1$. Then by [17, Thm B(ii)(a)] there is a subgroup $T\langle u\rangle \leq H$, with $T$ a maximal torus and $u$ a regular unipotent element of $\operatorname{SO}(V)$. We number the weights $\chi_{1}, \ldots, \chi_{2 l}$ of $T$ on $V$ such that $u$ acts as the permutation $(1,2, \ldots, 2 l-2)(2 l-1,2 l)$ on these. For $T_{1}=\operatorname{ker} \chi_{2 l-1} \cap \operatorname{ker} \chi_{2 l}$, the group $T_{1}\langle u\rangle$ is an example for case (2). On the other hand by taking the direct product of $\mathrm{GO}_{2}$ with a subgroup of $\mathrm{GO}_{2 l-2}$ as constructed in Example 7.5, and intersecting with $\mathrm{SO}(V)$ we find an example for (3), and as in part (1) we see that both lie inside proper parabolic subgroups.

(3) The example for $\mathrm{SO}_{6}=\mathrm{SL}_{4}$ in Proposition 7.2 falls into case (3); this can be seen from the weight spaces on the two modules, as the natural module for $\mathrm{SO}_{6}$ is the wedge square of the natural module for $A_{3}$. 
We are not aware of examples of torus normalisers in disconnected groups $A_{l} .2$ containing outer regular unipotent elements and lying in a proper parabolic subgroup.

7.4. Torus normalisers in simple exceptional groups. Finally, we investigate the case of exceptional groups.

Proposition 7.8. Let $T$ be a torus and $u \in \operatorname{Aut}(T)$ of prime-power order $p^{a}$. Then $\operatorname{dim} T \geq p^{a-1}(p-1)$.

Proof. We have $\operatorname{Aut}(T) \cong \mathrm{GL}_{n}(\mathbb{Z})$ with $n=\operatorname{dim} T$. If $u \in \mathrm{GL}_{n}(\mathbb{Z})$ has order $p^{a}$ then it must have an eigenvalue $\zeta$ that is a primitive $p^{a}$ th root of unity. But then all Galois conjugates of $\zeta$ are also eigenvalues of $u$, and there are $\varphi\left(p^{a}\right)=p^{a-1}(p-1)$ of these.

Remark 7.9. Let $T$ be a torus in a connected reductive group $G$ and $u \in G$ a unipotent element acting non-trivially on $T$. Then $p$ divides the order of the Weyl group of $G$. Indeed, by assumption $u \in N_{G}(T) / C_{G}(T)$ is non-trivial. As $L=C_{G}(T)$ is a Levi subgroup of $G$ and $N_{G}(T) \leq N_{G}(L)$, the claim follows with [15, Cor. 12.11].

Proposition 7.10. Let $G$ be simple of exceptional type and $X=T\langle u\rangle<G$ with $T$ a non-trivial torus and $u$ a regular unipotent element of $G$. Then one of the following holds:

(1) $G=E_{6}, p=3, \operatorname{dim} T=2$; or

(2) $G=E_{7}, p=2, \operatorname{dim} T=1$.

Proof. The regular unipotent element $u$ induces a non-trivial automorphism $\bar{u}$ of $T$, so by the previous remark, $p$ divides the order of the Weyl group of $G$.

Combining the $p$-power map on unipotent classes [6, Tab. D and E] and the structure of centralisers [9, $\S 22]$ we have compiled in Table 3 a list of the dimensions of maximal tori in the centralisers $C_{G}\left(u^{p^{i}}\right)$ for $i \geq 1$ and $u^{p^{i}} \neq 1$.

TABLE 3. Ranks of centralisers $C_{G}\left(u^{p^{i}}\right), i \geq 1$

\begin{tabular}{c|cccc} 
& $p=2$ & 3 & 5 & 7 \\
\hline$G_{2}$ & 0,1 & 0 & & \\
$F_{4}$ & $0,0,2$ & 0,3 & & \\
$E_{6}$ & $0,1,4$ & 2,5 & 2 & \\
$E_{7}$ & $1,2,4,6$ & 0,3 & 1 & 2 \\
$E_{8}$ & $0,1,2,4$ & $0,3,7$ & 0,7 & 1
\end{tabular}

Now first consider $G=G_{2}$. Then $\operatorname{dim} T \leq 2$, so $\bar{u}$ has order at most 4 when $p=2$, respectively 3 when $p=3$, by Proposition 7.8. Hence $u^{4}$, respectively $u^{3}$, must centralise $T$, which by Table 3 implies $\operatorname{dim} T=1$ and $p=2$. But in that case, $\bar{u}$ has order at most 2 , so $u^{2}$ centralises $T$ and we reach a contradiction to Table 3 ,

When $G=F_{4}$, then $\operatorname{dim} T \leq 4$ and by Proposition $7.8, \bar{u}$ has order at most 8 . Again by Table 3 this gives that $\bar{u}$ has order 8 and $\operatorname{dim} T \leq 3$, contradicting the bound in Proposition 7.8 .

For $G=E_{6}$ with $\operatorname{dim} T \leq 6$ we have $|\bar{u}| \leq 8,9,5$ when $p=2,3,5$ respectively. For $p=2$, using Proposition 7.8 and Table 3 we find that $\operatorname{dim} T=4$ and $\bar{u}$ of 
order 8 is the only possibility. Here $u^{8}$ lies in class $2 A_{1}$ by 6 , Tab. D], and its centraliser has rank 4 . Let $L$ be an $A_{1}^{2}$-Levi subgroup of $G$ containing $u^{8}$; it has connected centre $Z(L)^{\circ}$ of dimension 4 , so this must be the torus $T$ in $C_{G}\left(u^{8}\right)$. Now $u$ normalises $T$, so it also normalises $L=C_{G}(T)$, and thus $[L, L]=A_{1}^{2}$. If $u$ acts by an inner automorphism on $A_{1}^{2}$, then by Lemma 3.5 there is an element of order 16 centralising $A_{1}^{2}$, but the only elements of $G$ of that order are regular, a contradiction. Therefore, it acts by a graph automorphism on the $A_{1}^{2}$ and $u^{2}$ is inner and hence some element of order 8 centralises $A_{1}^{2}$. Again by [9] and [6] there is no element of order 8 in $G$ with such a centraliser. So this does not occur. Next, for the case $p=3$ using Proposition 7.8 and Table 3 as above, only $\operatorname{dim} T=2$ with $\bar{u}$ of order 3 remains. So $v:=u^{3}$, in class $D_{4}\left(a_{1}\right)$ by [6, Tab. D], centralises $T$, and we reach case (1) of the statement. The case $p=5$ is not possible by Table 3 .

For $G=E_{7}$ with Proposition 7.8 and Table 3 and arguing as above we are left with the case that $p=2$ and either $\operatorname{dim} T=4$ and $\bar{u}$ has order 8 , or $\operatorname{dim} T=2$ and $|\bar{u}|=4$, or $\operatorname{dim} T=1$ and $|\bar{u}|=2$. The last case occurs in the conclusion, so we need to exclude the former two. If $\operatorname{dim} T=2$ and $|\bar{u}|=4$, then $u^{4}$ centralises $T$ and lies in class $A_{4}+A_{1}$. Let $L$ be a Levi subgroup of this type containing $u^{4}$. It has centre $Z(L)^{\circ}$ of dimension 2 , so this is in fact $T$. Now $u$ normalises $T$ and hence also $[L, L]=A_{4} A_{1}$. Now $u^{2}$, of order 16 , acts as an inner element on this, and by Lemma 3.5 and using [6] and [9] we arrive at a contradiction. The case where $\operatorname{dim} T=4$ is similar.

Finally for $G=E_{8}$, the same line of argument as for the other groups shows that no new configurations occur.

Example 7.11. Both cases in Proposition 7.10 do actually lead to examples.

(a) Let $G=E_{6}$ with $p=3$. By [17, Thm A], there is a maximal subgroup $H=D_{4} T_{2} \cdot \mathfrak{S}_{3}$ of $G$ containing a regular unipotent element $u$, with $T_{2} \unlhd H$ a 2-dimensional torus. As $u^{3} \neq 1$ centralises $T_{2}$, the subgroup $T_{2}\langle u\rangle$ of $H$ then lies in a proper parabolic subgroup and so yields an example for the situation in Proposition 7.10(a).

(b) Let $G=E_{7}$ with $p=2$. According to [17, Thm A] there is a maximal subgroup $H=E_{6} T_{1} .2$ in $G$ containing a regular unipotent element $u$, with $T_{1} \unlhd H$ a 1-dimensional torus. Then $T_{1}\langle u\rangle \leq H$ yields an example for the situation in Proposition 7.10(b).

\section{ACKNOWLEDGMENTS}

This work was motivated in part by a question which Jay Taylor raised after a talk by the second author in Pisa. In addition, we acknowledge having had several useful conversations on cohomology with Steve Donkin, Jacques Thévenaz and Adam Thomas, and thank Thomas, Mikko Korhonen and David Craven for their careful reading of and comments on an earlier version, and David for spotting a gap in a proof.

\section{Note ADDED IN PROOF}

After becoming aware of our results, M. Bate, B. Martin and G. Röhrle in a recent preprint entitled "Overgroups of regular unipotent elements in reductive groups" have proposed a short, case-free proof of our Theorem 1 using the machinery of $G$-complete irreducibility. 


\section{REFERENCES}

[1] William W. Adams and Marc A. Rieffel, Adjoint functors and derived functors with an application to the cohomology of semigroups, J. Algebra 7 (1967), 25-34, DOI 10.1016/00218693(67)90065-8. MR218423

[2] Timothy C. Burness, Soumaïa Ghandour, Claude Marion, and Donna M. Testerman, Irreducible almost simple subgroups of classical algebraic groups, Mem. Amer. Math. Soc. 236 (2015), no. 1114, vi+110, DOI 10.1090/memo/1114. MR.3364837

[3] Timothy C. Burness and Donna M. Testerman, $A_{1}$-type subgroups containing regular unipotent elements, Forum Math. Sigma 7 (2019), Paper No. e12, 61, DOI 10.1017/fms.2019.12. MR.3942158

[4] Jens Carsten Jantzen, Representations of algebraic groups, 2nd ed., Mathematical Surveys and Monographs, vol. 107, American Mathematical Society, Providence, RI, 2003. MR2015057

[5] Peter Kleidman and Martin Liebeck, The subgroup structure of the finite classical groups, London Mathematical Society Lecture Note Series, vol. 129, Cambridge University Press, Cambridge, 1990, DOI 10.1017/CBO9780511629235. MR1057341

[6] R. Lawther, Jordan block sizes of unipotent elements in exceptional algebraic groups, Comm. Algebra 23 (1995), no. 11, 4125-4156, DOI 10.1080/00927879508825454. MR.1351124

[7] Martin W. Liebeck and Gary M. Seitz, Reductive subgroups of exceptional algebraic groups, Mem. Amer. Math. Soc. 121 (1996), no. 580, vi+111, DOI 10.1090/memo/0580. MR.1329942

[8] Martin W. Liebeck and Gary M. Seitz, The maximal subgroups of positive dimension in exceptional algebraic groups, Mem. Amer. Math. Soc. 169 (2004), no. 802, vi+227, DOI 10.1090/memo/0802. MR2044850

[9] Martin W. Liebeck and Gary M. Seitz, Unipotent and nilpotent classes in simple algebraic groups and Lie algebras, Mathematical Surveys and Monographs, vol. 180, American Mathematical Society, Providence, RI, 2012, DOI 10.1090/surv/180. MR2883501

[10] Martin W. Liebeck and Donna M. Testerman, Irreducible subgroups of algebraic groups, Q. J. Math. 55 (2004), no. 1, 47-55, DOI 10.1093/qjmath/55.1.47. MR2043006

[11] Alastair J. Litterick and Adam R. Thomas, Complete reducibility in good characteristic, Trans. Amer. Math. Soc. 370 (2018), no. 8, 5279-5340, DOI 10.1090/tran/7085. MR3803140

[12] Frank Lübeck, Small degree representations of finite Chevalley groups in defining characteristic, LMS J. Comput. Math. 4 (2001), 135-169, DOI 10.1112/S1461157000000838. MR1901354

[13] Gunter Malle, Generalized Deligne-Lusztig characters, J. Algebra 159 (1993), no. 1, 64-97, DOI 10.1006/jabr.1993.1147. MR.1231204

[14] Gunter Malle, Green functions for groups of types $E_{6}$ and $F_{4}$ in characteristic 2, Comm. Algebra 21 (1993), no. 3, 747-798, DOI 10.1080/00927879308824595. MR.1204754

[15] Gunter Malle and Donna Testerman, Linear algebraic groups and finite groups of Lie type, Cambridge Studies in Advanced Mathematics, vol. 133, Cambridge University Press, Cambridge, 2011, DOI 10.1017/CBO9780511994777. MR2850737

[16] George J. McNinch, Dimensional criteria for semisimplicity of representations, Proc. London Math. Soc. (3) 76 (1998), no. 1, 95-149, DOI 10.1112/S0024611598000045. MR.1476899

[17] Jan Saxl and Gary M. Seitz, Subgroups of algebraic groups containing regular unipotent elements, J. London Math. Soc. (2) 55 (1997), no. 2, 370-386, DOI 10.1112/S0024610797004808. MR 1438641

[18] Nicolas Spaltenstein, Classes unipotentes et sous-groupes de Borel (French), Lecture Notes in Mathematics, vol. 946, Springer-Verlag, Berlin-New York, 1982. MR672610

[19] T. A. Springer and R. Steinberg, Conjugacy classes, Seminar on Algebraic Groups and Related Finite Groups (The Institute for Advanced Study, Princeton, N.J., 1968/69), Lecture Notes in Mathematics, Vol. 131, Springer, Berlin, 1970, pp. 167-266. MR0268192

[20] David I. Stewart, The reductive subgroups of $F_{4}$, Mem. Amer. Math. Soc. 223 (2013), no. 1049, vi+88, DOI 10.1090/S0065-9266-2012-00668-X. MR3075783

[21] Irina D. Suprunenko, Irreducible representations of simple algebraic groups containing matrices with big Jordan blocks, Proc. London Math. Soc. (3) 71 (1995), no. 2, 281-332, DOI 10.1112/plms/s3-71.2.281. MR,1337469

[22] Donna Testerman and Alexandre Zalesski, Irreducibility in algebraic groups and regular unipotent elements, Proc. Amer. Math. Soc. 141 (2013), no. 1, 13-28, DOI 10.1090/S00029939-2012-11898-2. MR2988707 
[23] Donna M. Testerman and Alexandre E. Zalesski, Irreducible representations of simple algebraic groups in which a unipotent element is represented by a matrix with a single nontrivial Jordan block, J. Group Theory 21 (2018), no. 1, 1-20, DOI 10.1515/jgth-2017-0019. MR3739341

[24] Charles A. Weibel, An introduction to homological algebra, Cambridge Studies in Advanced Mathematics, vol. 38, Cambridge University Press, Cambridge, 1994, DOI 10.1017/CBO9781139644136. MR 1269324

FB Mathematik, TU Kaiserslautern, Postfach 3049, 67653 Kaiserslautern, Germany. Email address: malle@mathematik.uni-kl.de

Institut de Mathématiques, Station 8, École Polytechnique Fédérale de Lausanne, CH-1015 LAusanne, Switzerland

Email address: donna.testerman@epfl.ch 